\title{
Atomic and Electronic Structure of a Multidomain GeTe Crystal
}

Alexander S. Frolov ${ }^{1,2}$, Jaime Sánchez-Barriga ${ }^{3}$, Carolien Callaert ${ }^{4}, J$ oke Hadermann ${ }^{4}$, Alexander V. Fedorov ${ }^{3,5,6}$, Dmitry Yu. Usachov ${ }^{7}$, Alexander Chaika ${ }^{8}$, Brian Walls ${ }^{9}$, Kuanysh Zhussupbekov ${ }^{9}$, Igor V. Shvets ${ }^{9}$, Matthias Muntwiler ${ }^{10}$, Luca Gregoratti ${ }^{11}$, Matteo Amati $^{11}$, Andrei Varykhalov ${ }^{3}$, Oliver Rader ${ }^{3}$, Lada V. Yashina ${ }^{1,2, *}$

${ }^{1}$ Department of Chemistry, Moscow State University, Leninskie Gory 1/3, 119991 Moscow, Russia

${ }^{2}$ Semenov Federal research center for chemical physics, Kosygina str. 4, 119991 Moscow, Russia ${ }^{3}$ Helmholtz-Zentrum Berlin für Materialien und Energie, Elektronenspeicherring BESSY-II, Albert-EinsteinStr. 15, D-12489 Berlin, Germany

${ }^{4}$ EMAT, Department of Physics, University of Antwerp, Groenenborgerlaan 171, 2020 Antwerp, Belgium ${ }^{5}$ IFW Dresden, P.O. Box 270116, 01171 Dresden, Germany

${ }^{6}$ Joint Lab Functional Quantum Materials at BESSY-II, Albert-Einstein-Str. 15, D-12489 Berlin, Germany

${ }^{7}$ St. Petersburg State University, 7/9 Universitetskaya nab., St. Petersburg, 199034, Russia

${ }^{8}$ Institute of Solid State Physics RAS, Academician Ossipyan str. 2, Chernogolovka, 142432 Moscow

District, Russia

${ }^{9}$ CRANN, School of Physics, Trinity College Dublin, Dublin 2, Ireland

${ }^{10}$ Laboratory for Synchrotron Radiation - Condensed Matter, Paul Scherrer Institute, 5232 Villigen PSI, Switzerland

${ }^{11}$ Elettra - Sincrotrone Trieste S.C.p.A., Area Science Park, I-34012 Basovizza, Trieste, Italy

Abstract: Renewed interest in the ferroelectric semiconductor germanium telluride was recently triggered by the direct observation of a giant Rashba effect and a 30-year-old dream about a functional spin field-effect transistor. In this respect, all-electrical control
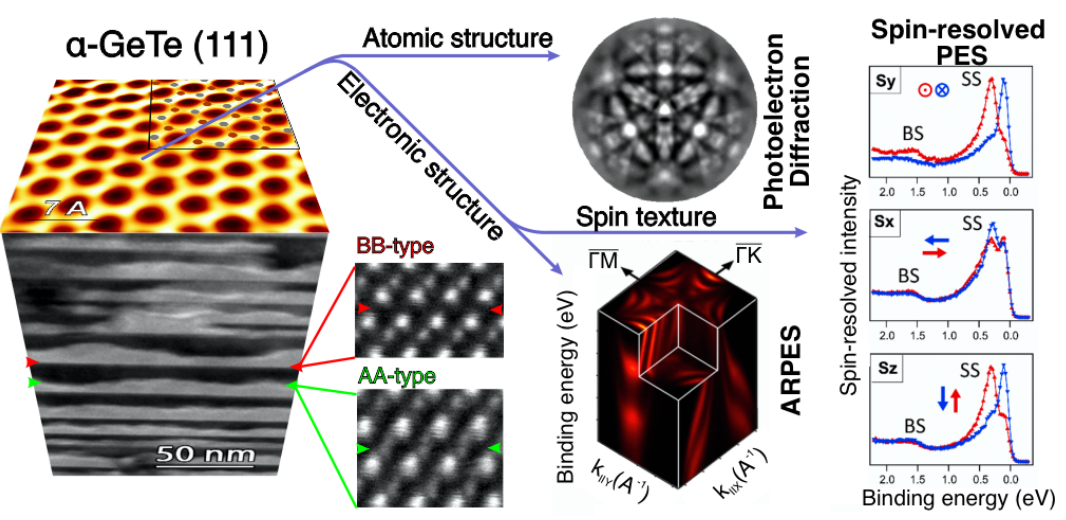
of the spin texture in this material in combination with ferroelectric properties at the nanoscale would create advanced functionalities in spintronics and data information processing. Here, we investigate the atomic and electronic properties of GeTe bulk single crystals and their (111) surfaces. We succeeded to grow crystals possessing solely inversion domains of $\sim 10 \mathrm{~nm}$ thickness parallel to each other. Using HAADF-TEM we observe two types of domain boundaries, one of them being similar in structure to the van-der-Waals gap in layered materials. This structure is responsible for the formation of surface domains with preferential Te termination $(\sim 68 \%)$ as we determined using photoelectron diffraction and XPS. The lateral dimensions of the surface domains are in the range of $\sim 10-100$ $\mathrm{nm}$, and both Ge and Te terminations reveal no reconstruction. Using spin-ARPES we establish an intrinsic quantitative relationship between the spin polarization of pure bulk states and the relative contribution of different terminations, a result which is consistent with a reversal of the spin texture of the bulk Rashba bands for opposite configurations of the ferroelectric polarization within individual nanodomains. Our findings are important for potential applications of ferroelectric 
Rashba semiconductors in non-volatile spintronic devices with advanced memory and computing capabilities at the nanoscale.

Keywords: germanium telluride, ferroelectric domains, Rashba effect, electronic structure, surface atomic structure, domain walls.

The spin field-effect transistor proposed by Datta and Das more than 30 years ago $^{1}$ paves the way for data information processing based on the electron spin. Together with the miniaturization of spintronic devices down to their ultimate size limit, their promising idea relies on the electrical manipulation of spin information transport without magnetic materials or external magnetic fields. ${ }^{2-4}$ To this end, the combination of ferroelectricity and spin-orbit coupling, such as in GeTe, represents an alternative route towards multifunctional devices with potential applications in future information technologies. $^{5}$

GeTe has been known for a long time as a narrow gap semiconductor and thermoelectric material. ${ }^{6-9}$ One of its unique properties is that it exhibits a reversible transition between crystalline and amorphous states which is also important for applications in non-volatile phase-change random access memories (PCRAMs). ${ }^{10}$ Recently, rhombohedral germanium telluride ( $a-G e T e)$ in single crystalline form has attracted a lot of attention as an intriguing material belonging to the class of ferroelectric Rashba semiconductors. ${ }^{5,11-15}$ In fact, $a-G e T e$ is one of the few known binary ferroelectric semiconductors with a narrow band gap. The chemical bond is less ionic and less covalent than that of its IV-VI analogs; this is the origin of rhombohedral distortion and spontaneous electric polarization of a-GeTe ascribed to an ordered Peierls dimerization. ${ }^{16}$ It is described based on the concept of resonant bonding ${ }^{17}$ from the fact that six bonds and three valent electrons per atom are in GeTe so that the $p$ electron density is highly delocalized and polarizable resulting in high optical dielectric constants. The unique combination of $p$-metallicity and ferroelectricity enables large spin-orbit splitting driven by an internal electric field through the Rashba effect. ${ }^{11-15}$ Indeed, a giant Rashba-type spin splitting of electronic states in GeTe was observed by spin- and angle-resolved photoemission (spin-ARPES) ${ }^{12-15,18}$ and it was shown that the spin polarization can be switched by applying an external voltage ${ }^{19}$ The relationship between the circulation of electron spins at the Fermi surface and the direction of the ferroelectric polarization was further investigated by spin-ARPES ${ }^{15}$ and ferroelectric order in single domains of few nanometers in size was observed by transmission electron microscopy (TEM) ${ }^{20}$ Consequently, GeTe is being considered as a unique platform for programmable spintronic devices with full-electrical switching.

In ferroelectrics, however, the switching process includes moving of domain walls. $^{21-23}$ At the nanoscale, a-GeTe exhibits a domain structure which can be different depending on the twinning plane. ${ }^{24}$ The most common structure is the so-called herringbone structure of hierarchical morphology. ${ }^{24,25}$ In such a multidomain structure, polarization reversal may involve intermediate steps, which lead to a deterioration of ferroelectric effects. ${ }^{26}$ Equally important, the atomic structure of domain boundaries, which can strongly influence the domain wall mobility, has not yet been studied precisely. 
Since the mechanism of ferroelectric repolarization is closely related to the properties of the domain structure, and the switching processes are highly influenced by the atomic structure of domain walls, engineering and manufacturing efficient GeTe-based spintronic devices requires deep understanding of the domain structure.

Up to date, previous photoemission studies on GeTe have been mainly focused on (111)-oriented films grown by molecular beam epitaxy (MBE). ${ }^{12-15,18,19}$ In these studies, the MBE grown films possess a single Te-terminated surface which is generally more stable. In this context, a surface-engineering strategy was recently put forward to access the inverted ferroelectric polarization of the Ge-terminated surface. ${ }^{15}$ In most of the cases, the studied films were assumed to be single domain, however, domains are clearly visible for films grown on InP substrates. ${ }^{19}$ Based on density-functional theory (DFT) calculations, ${ }^{15}$ it was shown that the surface electronic structure associated to Te and Ge terminations is different, with their corresponding Rashba-split surface states exhibiting opposite spin textures and lying below (above) the Fermi level for $\mathrm{Te}^{-}\left(\mathrm{Ge}^{-}\right)$terminated surfaces. Moreover, the spin splitting of bulk states is caused by the ferroelectric distortion, ${ }^{27}$ with the spin polarization pointing in opposite directions according the tendency of the Te-rich (Ge-rich) surface to display outwards (inwards) ferroelectric polarization. Experimentally, Ge-termination was not obtained for as-grown films, probably due to the lower surface energy associated to Te-termination. ${ }^{12}$ Therefore films grown in quasi-equilibrium conditions are expected to always possess single $\mathrm{Te}^{-}$ termination. Consequently, a pristine Ge-terminated surface is only possible to obtain by cleaving GeTe bulk single crystals, whose atomic and electronic properties have remained unexplored so far.

The cleavage plane for GeTe is exactly (111) and goes through longer Ge-Te bonds. Mono-domain single crystals of a-GeTe only exist in a scale of tens of nanometers due to the domain structure. ${ }^{24}$ To obtain flat surfaces, the structure should include solely inversion domains parallel to each other, which is not typical for GeTe. Generally, the domain structure can be controlled by deviation from stoichiometry and by heat treatment in the temperature range of the cubic-to-rhombohedral phase transition.

In this work, we investigate the influence of the domain structure of a-GeTe single crystals with parallel inversion domains on the atomic and electronic structure of the (111) surface. To this end, the crystals are cleaved in situ and characterized using a complementary set of the modern experimental tools, including atomic resolution scanning transmission electron microscopy (HAADF-STEM) combined with energy dispersive X-ray analysis (STEM-EDX), scanning tunneling and atomic force microscopy (STM/AFM), ARPES and spin-ARPES, X-ray photoemission spectroscopy (XPS) and Xray photoelectron diffraction (XPD).

We show that in the case of inversion domains there are two type of boundaries: flat Te-Te boundaries of the van der Waals (vdW) type and non-planar boundaries of more complex structure including a corrugated Ge layer. The natural cleavage plane proceeds not only between Te-Te layers but also though domains of different polarity, resulting in surface domains of both $\mathrm{Te}$ and Ge terminations in the ratio of 68/32. Both terminations reveal no reconstruction. The Te-terminated surface shows minor relaxation of $\sim 3 \%$ and a surface core level shift (SCLS) in the Te $4 d$ spectra 
corresponding to the potential due to the internal polarization of the top domains. Band structure study reveals surface states associated to Te termination that exhibit large spin splitting and high degree of spin polarization, but also previously unmeasured bulkderived surface resonances up to high binding energies as well as bulk states with reduced spin polarization, the magnitude of which is intrinsically related to the relative contribution of both Te and Ge terminations in a quantitative way. Finally, the surface domains of different termination could be a platform for the development of multifunctional spintronic devices, where the electrical reconfiguration of the information encoded by the electron spin could be exploited, for instance, in non-volatile ultrahigh density data storage applications.

\section{Results and discussion}

\section{Bulk domain structure and atomic structure of domain boundaries}

As grown GeTe during cooling undergoes a martensite-type phase transition at 630-700 K (depending on its stoichiometry) from the rock salt $F m \overline{3} m$ structure ( $\beta$-GeTe) to the rhombohedral $R 3 \mathrm{~m}$ structure $(\alpha-\mathrm{GeTe})$ stable at room temperature, where one of the cubic $[111]_{\mathrm{c}}$ directions becomes the polar rhombohedral $[111]_{\mathrm{R}}$ or the hexagonal $[001]_{\mathrm{H}}$ direction. ${ }^{24}$ A relative displacement of the Ge and Te sublattices along the $[111]_{\mathrm{R}}$ direction makes the planar distances between successive (111) Ge and (111) Te planes unequal as $1.48 \AA$ and $2.15 \AA$, with the corresponding Ge-Te bond lengths of $2.84 \AA$ and $3.15 \AA .{ }^{28}$ As a consequence of this transformation, the crystal becomes noncentrosymmetric. The rhombohedral crystals always contain domains with different orientations. Different angles between domains appear depending on the mutual orientation of the corresponding (111) type planes: $71^{\circ}, 109^{\circ}$ and $180^{\circ}$. Possible domain wall configurations are schematically presented in Fig. 1. According to Snykers et al., ${ }^{24}$ domains can be organized as reflection twins showing head to head coupling (Fig. 1b,d) along (100) and (110) planes and rotation twins with head to tail coupling along (100) and (110) planes (Fig. 1c,e). They form the so-called herringbone structure which can be revealed by microscopy. ${ }^{25}$ Inversion boundaries of planar arrangement are formed by rotation twins, when the boundary is parallel to the trigonal axis, and reflection twins with the boundary perpendicular to the trigonal axis. From a common structural consideration for the latter case four types of domain walls are possible with two shorter distances around Ge or Te and with two longer distances around Ge or Te (Fig. 1g-j). Thus, in Fig. $1 \mathrm{~g}$ and $\mathrm{h}$, the center layer (Te/Ge) appears «overbonded», and the flanking (Ge/Te) "underbonded», whereas in Fig. $1 \mathrm{i}, \mathrm{j}$ the center layer is "underbonded» in its entirety. Therefore, Snykers model is of fully geometrical nature and considers unrelaxed bonds without taking into account the chemistry of constituent elements. It mostly aims at explaining the possible mutual orientation of different domains rather than atomic structure of domain walls. The atomic structure of domain wall is in fact a subject of detailed investigation as described above.

Generally, $\alpha-G e T e$ crystals possess a domain structure of the herringbone type. Meanwhile, the appearance of different domain structures depends on the deviation from 
stoichiometry and post-growth temperature regime. This gives insight on how to control the resulting domain arrangement on the surface.

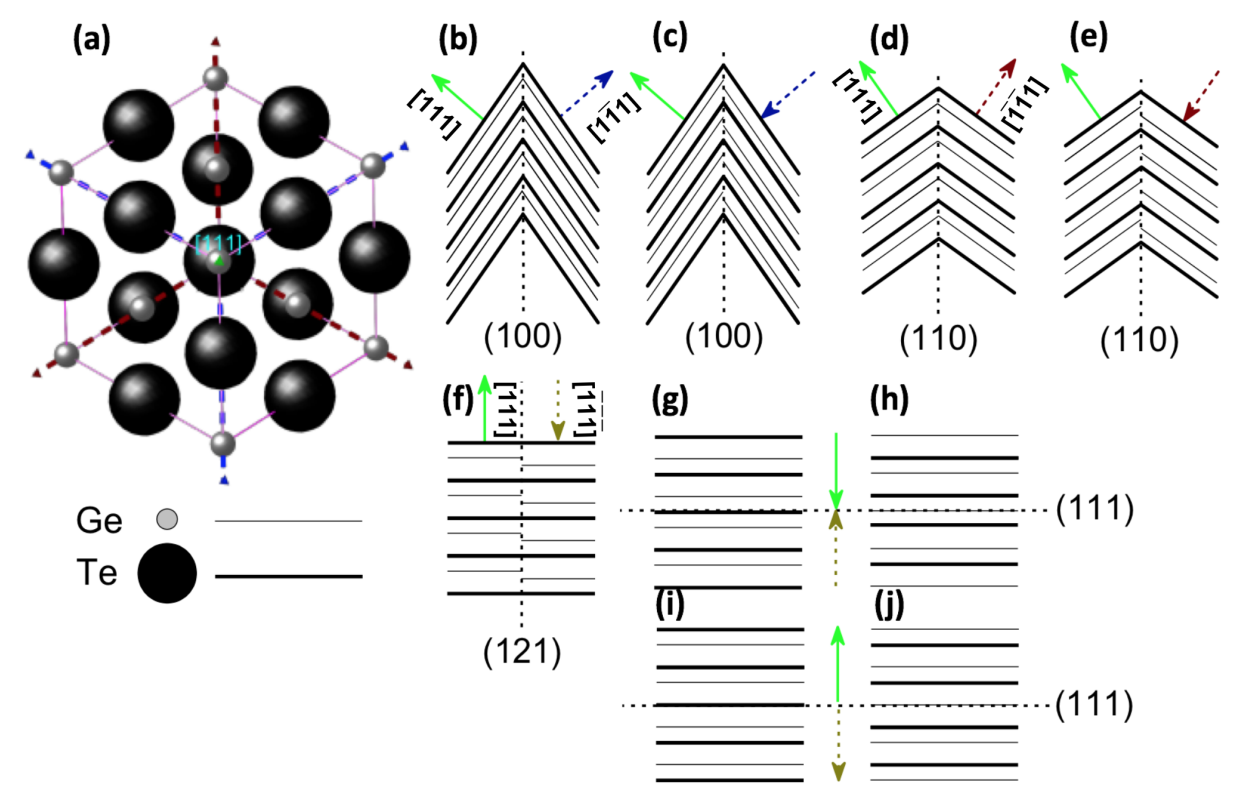

Figure 1. (a) Atomic structure of high-temperature cubic GeTe with the directions of the rhombohedral distortion. (b)-(e) Schematic representation of mutual domain orientation with different twining plane. (f)-(j) Different possibilities of the inversion domain stacking.

For our crystals, solely inversion domain boundaries perpendicular to $[111]_{\mathrm{R}}$ are observed. They are clearly seen from the zeroth order bright-field TEM image and darkfield TEM image (using reflections $003_{\mathrm{H}}$ and $006_{\mathrm{H}}$ ) of the same area taken along the zone axis $[2-1-1]_{R}$ (Figs. 2a,b).

In bright field mode, successive domains with inversion domain boundaries are observed to have the same background contrast independent of the diffraction conditions. In dark field mode, the domains have opposite contrast (dark-bright) under multiple beam conditions and the specific selection of the active reflections. ${ }^{24}$ In both bright- and dark field modes we clearly observe an alternation of sharp and wavy (stepwise) interfaces perpendicular to $[111]_{R}$, which we identify as the AA and BB interfaces, respectively. Both $\mathrm{AA}$ and $\mathrm{BB}$ are inversion domain boundaries, which appear due to the loss of the inversion symmetry upon the phase transition. The medium distance between two BB domain boundaries is $12.3 \mathrm{~nm}$ with an average of $13.6 \pm 7 \mathrm{~nm}$. Snykers et al. ${ }^{24}$ and Lee et al. ${ }^{25}$ previously detected inversion domain boundaries in GeTe in more complex configurations like herringbone structures, which differ from our situation.

Further investigation of the domain boundaries with HAADF-STEM uncovers that the atomic structure of both type of interfaces is different from those we derive based on the common structural consideration discussed above. In more detail, Figs. 2c,f,g evidence that the BB boundaries are atomically flat while the stepwise AA interfaces go through different layers. These twin boundaries are similar to twinning dislocations, a term used for deformation twins. ${ }^{31}$ These boundaries perpendicular to the $[001]_{\mathrm{H}}$ direction cause streaks along the $\mathrm{c}_{\mathrm{H}} *$ axis in the corresponding diffraction patterns shown in Fig. 2f. 

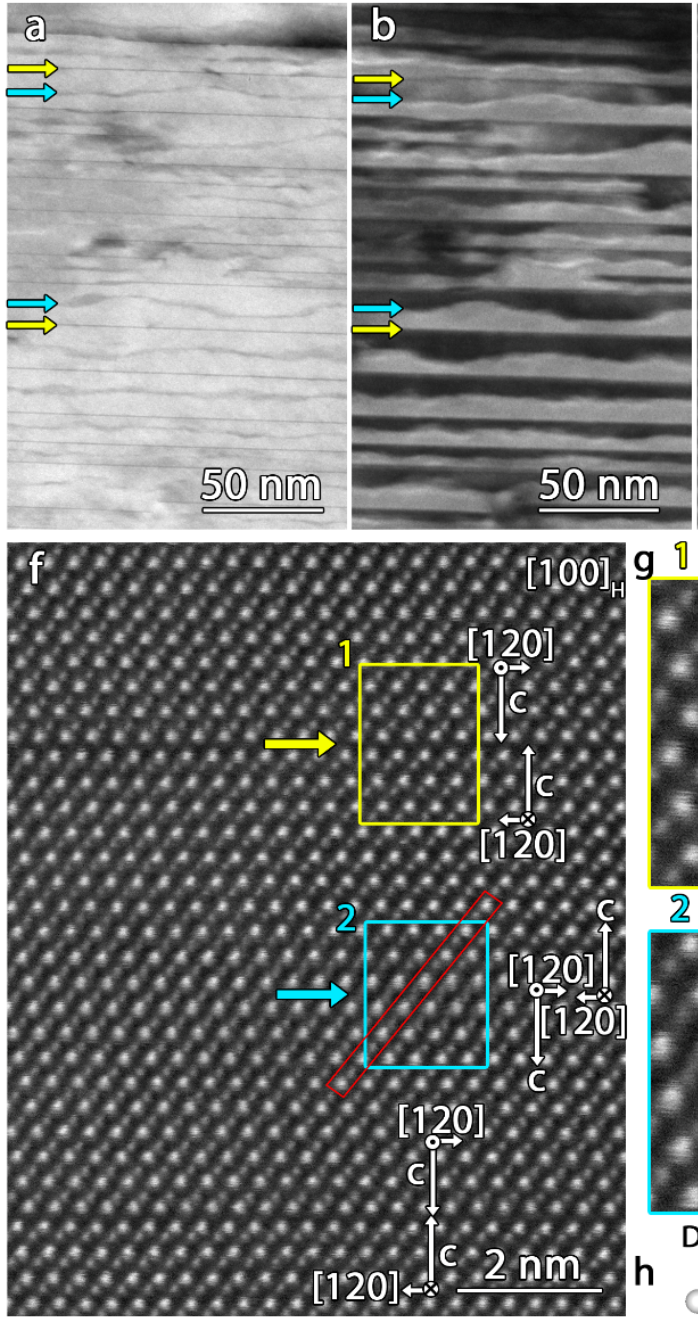
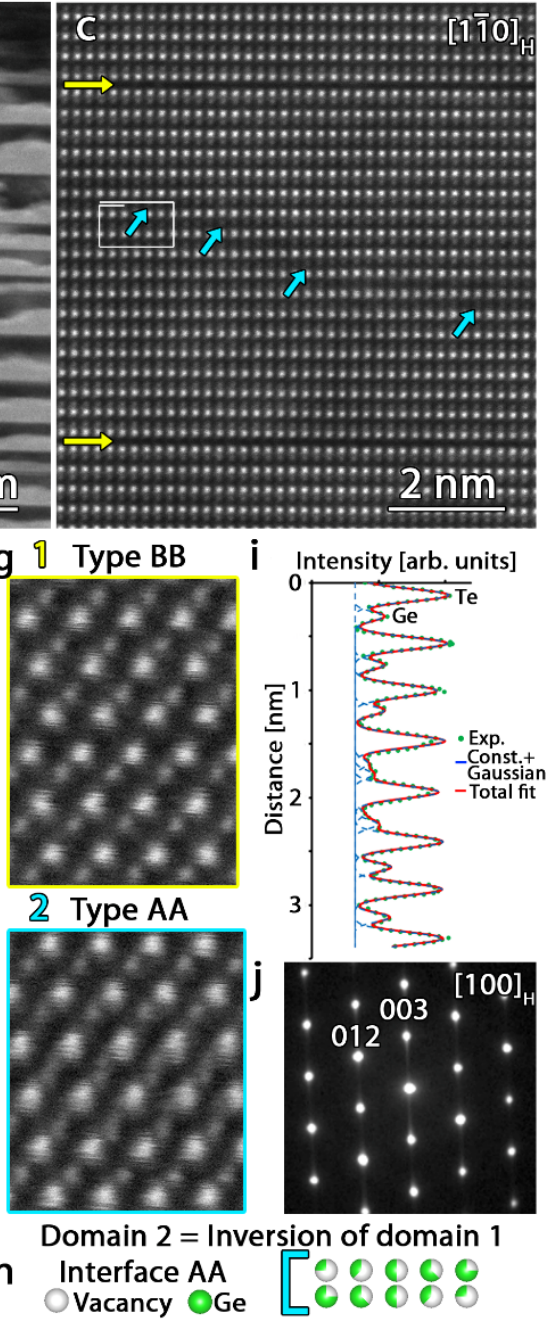

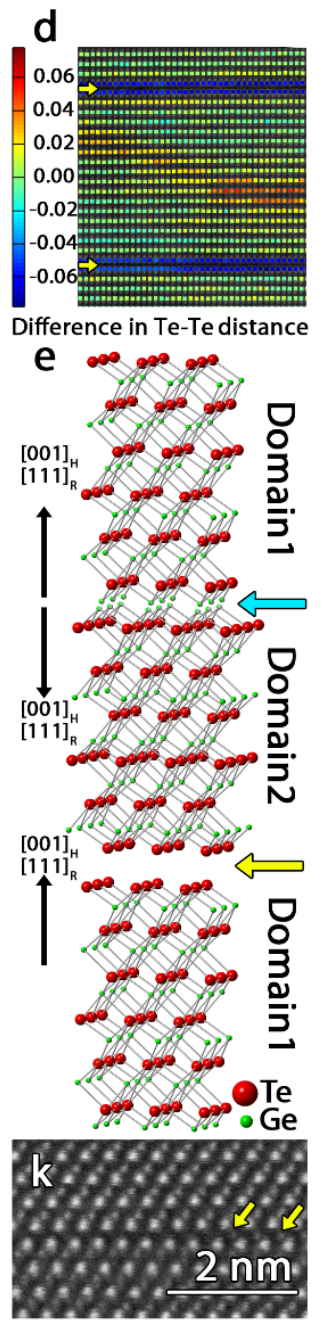

Figure 2. (a) Zeroth order bright-field TEM image and (b) corresponding dark-field TEM image, while selecting the $003_{\mathrm{H}}$ and $006_{\mathrm{H}}$ reflections, of zone $[2-1-1]_{\mathrm{R}}=[1-10]_{\mathrm{H}}$ where a couple of type BB (AA) domain boundaries are marked with yellow (blue) arrows. (c) Averaged HAADF-STEM image of zone $[2-1-1]_{\mathrm{R}}$ using a short frame time $(2.52 \mathrm{~s})$ to limit distortions, showing a sharp BB interface in a single layer (yellow arrows) and a stepwise AA interface (blue arrows) with a marked Burgers vector. (d) Strain map of the HAADF STEM image in (a) calculated with StatSTEM ${ }^{29}$ using the $\mathrm{Te}$-Te interlayer distances projected onto the $\mathrm{c}_{\mathrm{H}}$ direction. Positive values indicate an increase in the projected interlayer $\mathrm{Te}-\mathrm{Te}$ distance relative to the distance in the undistorted parent compound, negative values a decrease. (e) Our proposed model for the domain structure. (f) HAADF-STEM image of zone $[1-10]_{\mathrm{R}}=[100]_{\mathrm{H}}$ with type BB (yellow) and type AA (blue) interfaces, where $(\mathrm{j})$ shows a diffraction pattern of the corresponding zone with slight streaks along the $\mathrm{C}_{\mathrm{H}}$ * axis caused by the domain boundaries perpendicular to the $[001]_{\mathrm{H}}$ direction. (g) Enlargement of the two areas in (f). (h) Parallel to (001), a gradual occupancy transition occurs at the AA interface between the two Ge rows (schematic representation). (i) Line profile of the red area in (f), fitted with Fityk, ${ }^{30}$ showing the experimental data (green dots), the total fitted curve (red line) and the separate Gaussian fits with a constant background (blue striped line). At the AA interface, the two separate Ge positions between the Te layers have clearly a lower intensity, which supports the gradual occupancy transition. (k) Interstitial Ge atoms inside the BB interface. 
Figure $2 \mathrm{~d}$ shows the strain map calculated from the interatomic distances in the HAADF-STEM images. The relative shift of the Ge and Te sublattices along the $[111]_{\mathrm{R}}$ direction, which occurs in opposite senses in neighboring domains, explains the observed expansion and compression at the interface. It is clearly seen that the flatter part of the AA boundary has higher tensile strain. In the projection at the expanded AA interface, either a centered Ge site or two Ge sites between the Te layers are visible in the HAADFSTEM images. Note that these images are projections of the structure over the whole atom column and in every unit cell only one of two Ge positions at the AA interface can be occupied due to geometric restrictions (Fig. 1c,d,f,h). Lee et al. ${ }^{25}$ observed an expansion strain at this interface, but suggest a centered Ge atom. In their case, however, the domain boundary is $5 \mathrm{~nm}$-thick, while the size of our domain boundaries is at the atomic scale.

In contrast to the AA boundary, a uniform compressive strain is calculated around the BB boundary and is shown in Fig, 2d. This strain can be released by pushing Ge atoms out or, in other words, by the accumulation of cation vacancies, which leads to two consecutive (111) Te planes. Such vacancies are available and always present in GeTe since its equilibrium homogeneity range is totally shifted to the Te-rich side, so the vacancy concentration can reach 1.5 at.\% giving a hole concentration from $10^{19}$ to $10^{21} \mathrm{~cm}^{-3}$ for $\mathrm{Ge}^{-}$and $\mathrm{Te}-$ boundaries, respectively. ${ }^{32-35}$ Our samples are Te-enriched and, therefore, the bulk density of vacancies is sufficient to form BB interfaces with mean spacing of $\sim 10 \mathrm{~nm}$. The BB interface seems to be similar to the $\mathrm{vdW}$ gap in layered $\mathrm{Bi}_{2} \mathrm{Te}_{3} \cdot{ }^{36,37}$ The decisive role of vacancies in the formation of such interfaces is supported by the fact that the domain structure with planar inversion domain is favored for Te-rich GeTe. This agrees with Sist et al. ${ }^{35}$ who speculated the possibility of vacancy accumulation at domain boundaries. It should be noted that non-random vacancy distribution such as the BB interface occurs due to the necessity of local charge neutrality, and leads to extended defects also in some other materials such as e.g. $\mathrm{TiO}_{\mathrm{x}}$, $\mathrm{NbO}_{x}$ Magneli phases ${ }^{38,39}$.

The structure of $\mathrm{BB}$ domain boundaries implies their lower mobility and, hence, slower switching than those for Snykers model (Fig. 1h), since this involves atomic motion from one layer to another. More examples of HAADF-STEM images can be found in Supporting information (Figs SI1 - SI4).

\section{Surface structure and termination}

Planar configuration of the inversion domains enables a large quasi-flat cleavage plane corresponding to the [111] direction. However, the SEM and AFM images in Fig. 3a,b reveal that the surface has a relatively high roughness. The step height is in the nm range and comparable with but generally smaller than the typical domain thickness as it directly follows from Fig 3c. Therefore, the cleavage plane unavoidably proceeds through domains of different polarity i.e., not only along BB boundaries presenting vdWlike gaps. The lateral dimensions of surface domains are in the range of tens to hundreds of nanometers as it follows from our AFM and STM measurements. Within flat areas, atomic steps with a height corresponding to a Ge-Te (111) bilayer $(0.36 \mathrm{~nm})$ or 2 bilayers 
are typically observed. Examples are given in Figs. 3e-h. In very rare cases, true atomic steps of about $0.17-0.20 \mathrm{~nm}$ can be distinguished. These steps are likely related to the AA boundaries since the cleavage plane proceeds through the breaking of longer Ge-Te bonds.
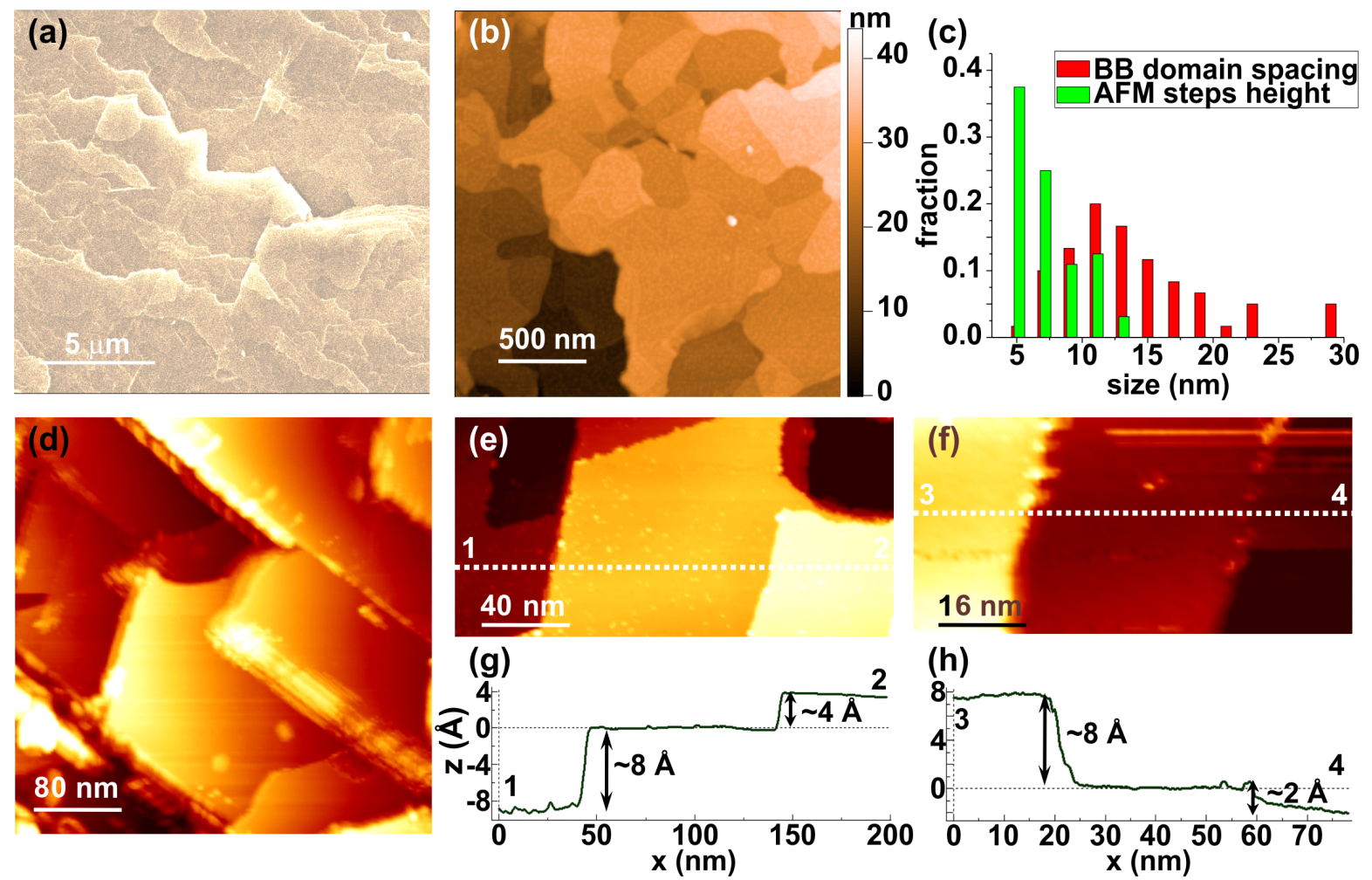

Figure 3. Steps at GeTe (111) cleavage surface: (a) as measured by SEM (cleaved in air), (b) AFM image (cleaved in air), (c) step height distribution (green, from AFM) and BB domain spacing (red, from TEM). (d) STM image of clean surface, (e), (f) atomic steps revealed by STM and (g),(h) corresponding height profiles along the white dashed lines shown in (e),(f). The STM images were measured at $\mathrm{U}=-3.0 \mathrm{~V}$ and $\mathrm{I}=40 \mathrm{pA}(\mathrm{d}), \mathrm{U}=2.5 \mathrm{~V}$ and $\mathrm{I}=25 \mathrm{pA}(\mathrm{e})$, and $\mathrm{U}=2.2 \mathrm{~V}$ and $I=45 \mathrm{pA}(\mathrm{f})$.

Apart from steps we observed two types of atomic scale defects: (i) dark depressions with three-fold symmetry which are better seen in the images measured at large bias voltages and (ii) bright protrusions which are resolved in all the range of bias voltages (see also Figs. S5 and S6 of the Supporting information). The defect density is about $2-3 * 10^{8} \mathrm{~cm}^{-2}$. Additional measurements performed under different conditions further support that bright defects correspond to adatoms, while dark depressions are related to subsurface defects in the GeTe lattice. It should be mentioned that the surface areas with increased number of bright protrusions are evidently related to the more chemically reactive Ge-termination (Fig. S5b).

More insight on the atomic structure of the surface domains is provided in Fig. 4. Both LEED (Fig. 4a) and STM measurements (Fig. 4b,c) demonstrate six-fold symmetry with an atomic spacing corresponding to the $\mathrm{Ge}-\mathrm{Ge}$ (or $\mathrm{Te}-\mathrm{Te}$ ) distance in the layer (Fig. 4b) or a honeycomb pattern (Fig. 4c). The latter is recorded at the same surface domain and fits well to the joint atomic motif of the very surface layer and the next underlying atomic layer. The appearance of two different patterns depends not only on 
the bias voltage but also on the tip-to-surface distance, tunnelling gap resistance and tip state. It should be mentioned that we obtained atomic resolution exclusively at relatively low tip-to-surface distances, and therefore, the influence of the tip state is rather high. As a consequence, the two different patterns measured from the same surface area can be explained by strong tip-to-surface interaction at very small tunneling gaps, which lead to the modification of the surface atom electronic structure ${ }^{40}$ and to substantial relaxations of the surface atoms during scanning ${ }^{41}$. Different relaxations of Ge and Te atoms interacting with the W[001] tip atom enable this situation when both sublattices of the GeTe (111) surface bilayer are visualized by STM. All in all, both LEED and STM clearly evidence the $(1 \times 1)$ ordering and the absence of a structural reconstruction as predicted theoretically by DFT modelling. ${ }^{42}$
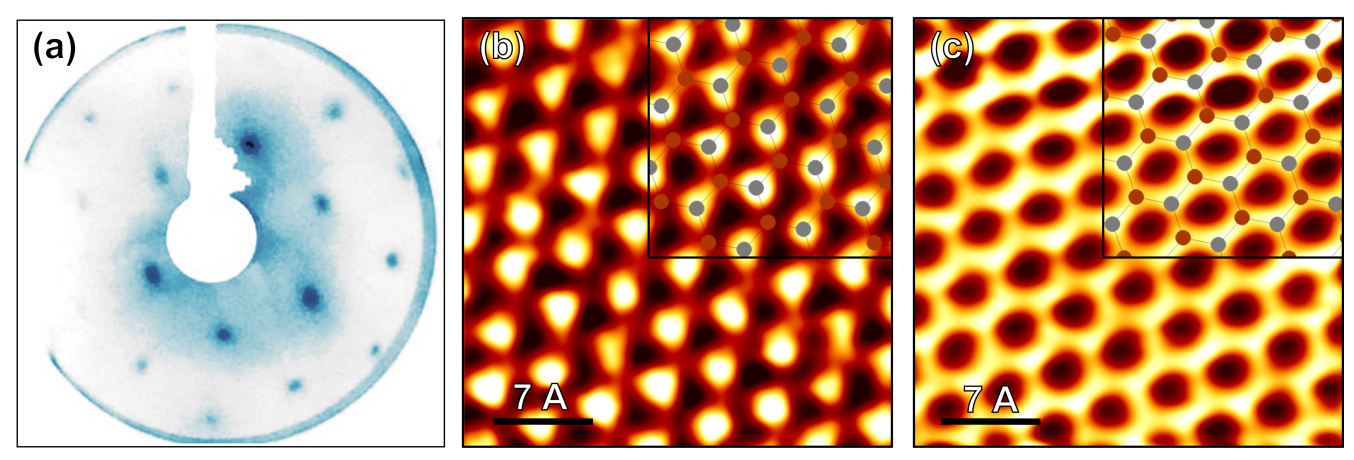

Figure 4. Atomic structure of the GeTe (111) surface: (a) LEED pattern measured at $60.6 \mathrm{eV}$, (b), (c) STM images obtained at approximately the same tunneling gap resistance but different tip states. The STM images were measured at $\mathrm{U}=0.5 \mathrm{~V}$ and $\mathrm{I}=40 \mathrm{pA}(\mathrm{b})$, and $\mathrm{U}=0.45 \mathrm{~V}$ and $\mathrm{I}=30 \mathrm{pA}$ (c). The images are shown with the GeTe lattice overlaid. Grey and red circles show the positions of Te and Ge atoms in the top bilayer (based on the bulk structure).

To obtain further insight into both surface terminations and their atomic structure, we used photoelectron diffraction - a method sensitive to the local structure around an atom of interest. ${ }^{43,44}$ The corresponding Ge $3 d$ and Te $4 d$ diffraction patterns are shown in Fig. 5. It should be emphasized that for a Te-terminated surface Ge atoms have 3 Te atoms above with shorter distance whereas for a Ge-terminated surface Ge atoms (in the third layer) have 3 Te atoms above with longer distances. By analogy, each Te atom for a Ge-terminated surface has $3 \mathrm{Ge}$ atoms above with shorter distances whereas for a Teterminated surface Te atoms (in the third layer) have $3 \mathrm{Ge}$ atoms above with longer bond lengths. This gives rise to the similarity between the simulated Ge $3 d$ pattern corresponding to the Te-terminated surface in Fig. 5e and the simulated Te $4 d$ pattern corresponding to Ge-terminated surface in Fig. 5h. Very clearly, the experimental diffraction patterns for both core levels can only described properly if a combination of the structures corresponding to Ge and Te terminations are considered, a result which is again consistent with the formation of a natural multidomain structure related to the two terminations in absence of surface reconstruction. Optimization plots for both patterns presented in Fig. 5e correspond to the Ge-termination covers $32 \pm 8 \%$ of the total area. The error was estimated as described in Ref. ${ }^{45}$ In addition, the first interatomic distance 
was optimized for both terminations resulting in a negligible contraction of $1.5 \%$ for Te and $0.5 \%$ for Ge.

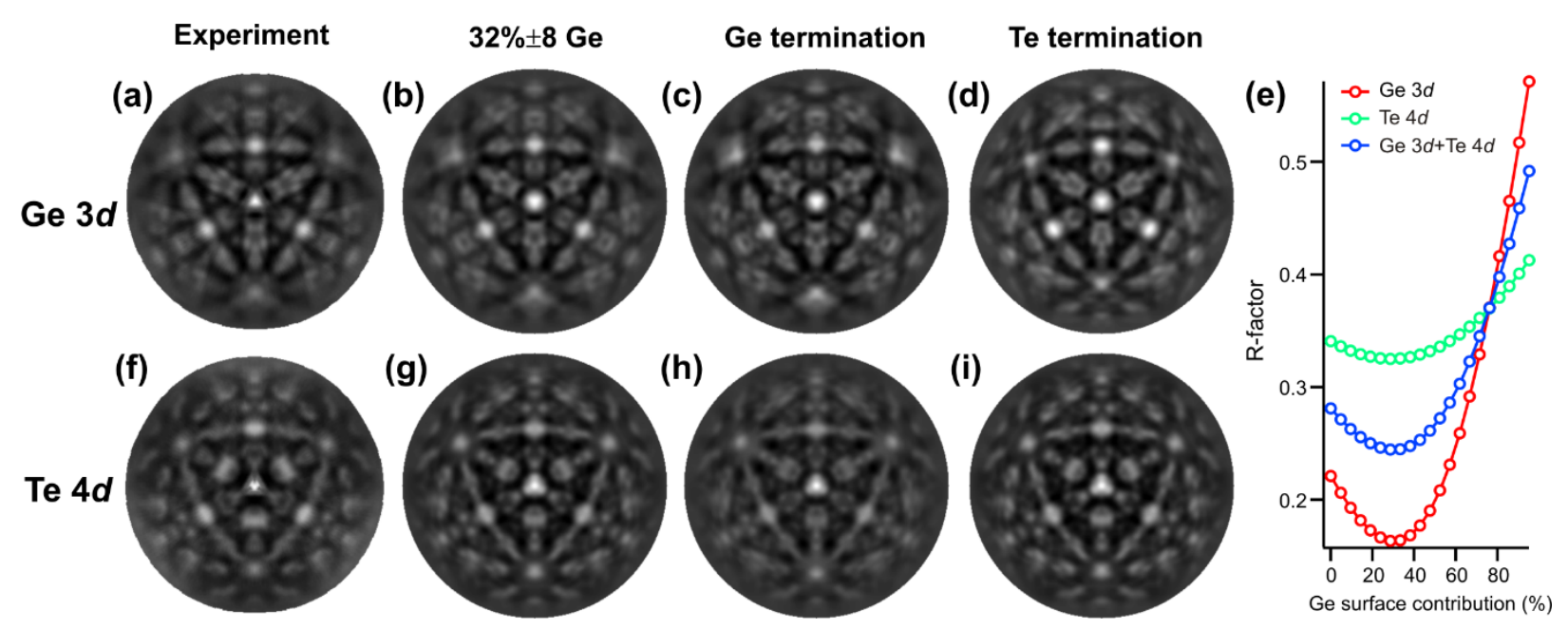

Figure 5. Photoelectron diffraction patterns of GeTe (111) A+B surface. (a)-(d) Ge 3d and (f)-(i) Te 4d diffraction patterns at electron kinetic energies of 565 and $555 \mathrm{eV}$, respectively. (a),(f) Experimental diffraction patterns. (b)-(d) and (g)-(i): Corresponding simulations for (b), (g) mixed, (c),(h) Ge and (d),(i) Te terminations. (e) R-factor dependence on the contribution of the Geterminated surface.

High-resolution XPS measurements and DFT calculations shown in Fig. 6 reveal that for the shallow Te $4 d$ core level the photoemission peak has at least two components split by $-0.38 \mathrm{eV}$, with their intensity ratio depending on the probing depth (Figs. 6d). More in detail, in the kinetic energy range of 50-400 eV the intensity of the low binding energy (BE) component decreases evidencing its surface nature (see Fig. S7 of the Supporting information). Similarly, the Ge $3 d$ peak in Fig. 6c cannot be described by a single component, however in this case the intensity ratio of individual components has no pronounced dispersion as a function of kinetic energy.

To model core level shifts we calculated 12 -layer slab having both $\mathrm{Te}-$ and $\mathrm{Ge}^{-}$ termination from different sides (first layer corresponds to Ge termination and twelfth layer is Te termination). According to Fig. 6a both Te and Ge terminations undergo surface relaxation which involves a slight contraction of the first (short) interlayer distance and an increase of the second (long) interlayer distance by about $3 \%$. This agrees qualitatively to the XPD data described above. Besides, the effective charge for Te or Ge atoms is different for the atoms located within different layers due to the outwards polarization $P_{\text {out }}$ of the Te-terminated surface or the inwards polarization $P_{\text {in }}$ of the $\mathrm{Ge}^{-}$ terminated surface. This is illustrated in Fig. 6b. For that reason, surface Te atoms bear a charge different from that of the second Te layer according to the calculation. This causes the negative surface core-level shift of $-0.34 \mathrm{eV}$ that explains our experimental observations. The shift for each layer is different, therefore, we model theoretical corelevel spectra for $\mathrm{Te}$ - and Ge-terminated surfaces taking into account not only the surface sensitivity but also the contribution of each individual layer. The partial contributions are shown in Fig. 6e-h. Both Te $4 d$ and Ge $3 d$ spectra can be qualitatively described by considering mixed contributions from both the $\mathrm{Te}^{-}$and $\mathrm{Ge}^{-}$-terminated surface, as shown 
in Figs. 6c,d. In particular, from the results of Fig. 5 and 6 taken altogether, we derive relative contributions of $68 \pm 8 \%$ and $32 \pm 8 \%$ from Te and Ge termination, respectively. It should also be noted that for a GeTe surface exhibiting a herringbone domain configuration mentioned above, the spectral shape would be different from the one seen in Figs. 6c,d as it was measured in Ref. ${ }^{46}$. In addition, the experimental SCLS of $-0.38 \mathrm{eV}$ in Te $4 d$ spectra serves as indication for the Rashba effect occurring at the surface.

(a)

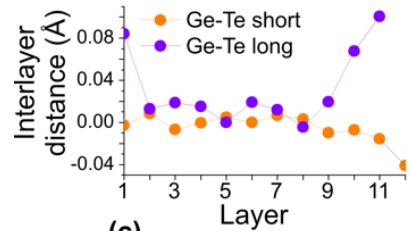

(c)

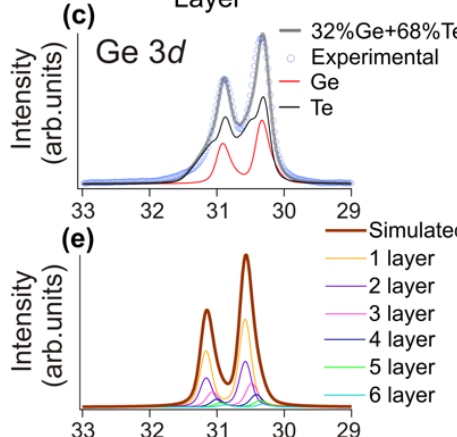

(g)

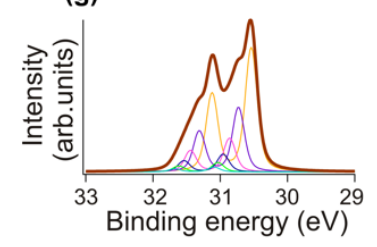

(b)

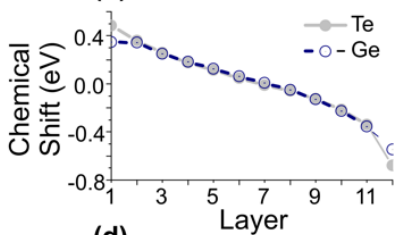

(d)

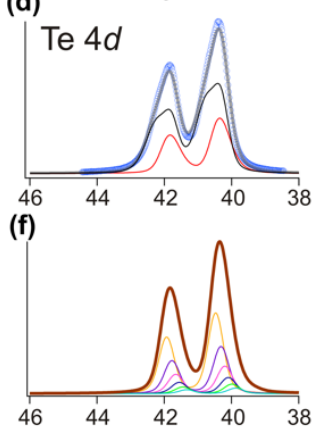

(h)

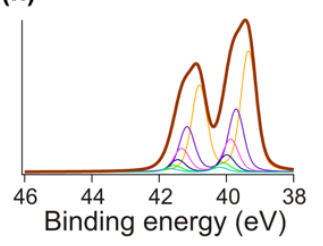

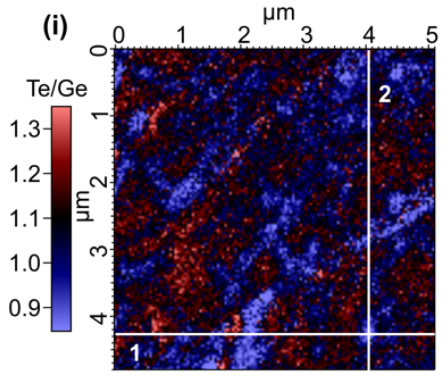

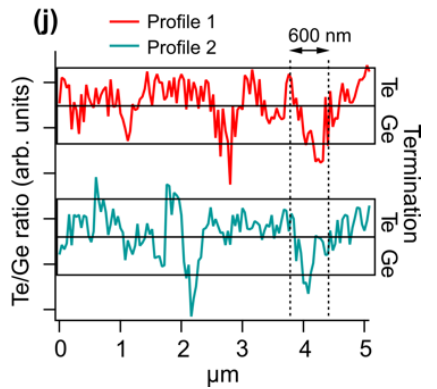

Figure 6. Core-level spectra of GeTe (111) A+B surface. (a) Layer-resolved DFT calculation of the interlayer distances for short and long Ge-Te bonds (relative to simulated bulk distances) for a 12-layer slab modelling both terminations. (b) Calculated core-level shifts for the different layers of the same slab. (c), (d) Comparison between experimental and DFT calculated core-level spectra for (c) Ge $3 d$ and (d) Te $4 d$ core-levels. The theoretical spectra correspond to a mixed termination of $32 \% \mathrm{Ge}$ and $68 \% \mathrm{Te}$. All experimental and theoretical spectra were obtained with a fixed kinetic energy of $50 \mathrm{eV}$ for Ge $3 d_{5 / 2}$ and Te $4 d_{5 / 2}$. (e)-(h) Separated contributions from the different layers in the calculated spectra of (e),(g) Ge $3 d$ and (f),(h) Te $4 d$ core levels for (e), (f) $\mathrm{Ge}^{-}$and (g),(h) Te-terminated surfaces. (i) Spectromicroscopy map showing Ge to Te ratio as obtained from Ge 3d and Te 4d intensity maps (for more details see Supporting Information file). (j) Corresponding line profiles as extracted from the vertical and horizontal lines labeled 1 and 2 in (i). 
Direct mapping of surface composition is shown in Fig.6 h. In detail, the Ge/Te ratio is displayed after background subtraction as described in the Supporting Information file. From this result we estimate that under our experimental conditions the Ge/Te ratio varies from 1.21 for Ge-termination to 0.82 for Te-termination. This fits well to our experimental observations indicating the presence of tens to hundreds $\mathrm{nm}$ areas of different terminations, that is fully in line with the AFM data discussed above.

\section{Electronic and spin structure}

To further understand the influence of the multidomain structure on the electronic properties, we performed ARPES measurements at different photon energies as shown in Figs. 7 and 8. It is understood that the size of the synchrotron light spot $(>100 \mu \mathrm{m})$ largely exceeds the lateral dimensions of the surface nanodomains and, thus, by ARPES we probe a mixture of both Te and Ge terminations. If we now compare the energy-momentum dispersions measured at $25 \mathrm{eV}$ (Figs. 7a-e) and $21 \mathrm{eV}$ (Figs. 7f-j), we identify distinct surface and bulk-related features, the character of which can be clearly recognized because pure bulk states display a strong photon energy dependence or $\mathrm{k}_{\mathrm{Z}}$ dispersion, ${ }^{47,48}$ as evidenced when comparing Figs. $7 \mathrm{~b}$ and $7 \mathrm{~g}$ as well as from the results in Fig. 8.
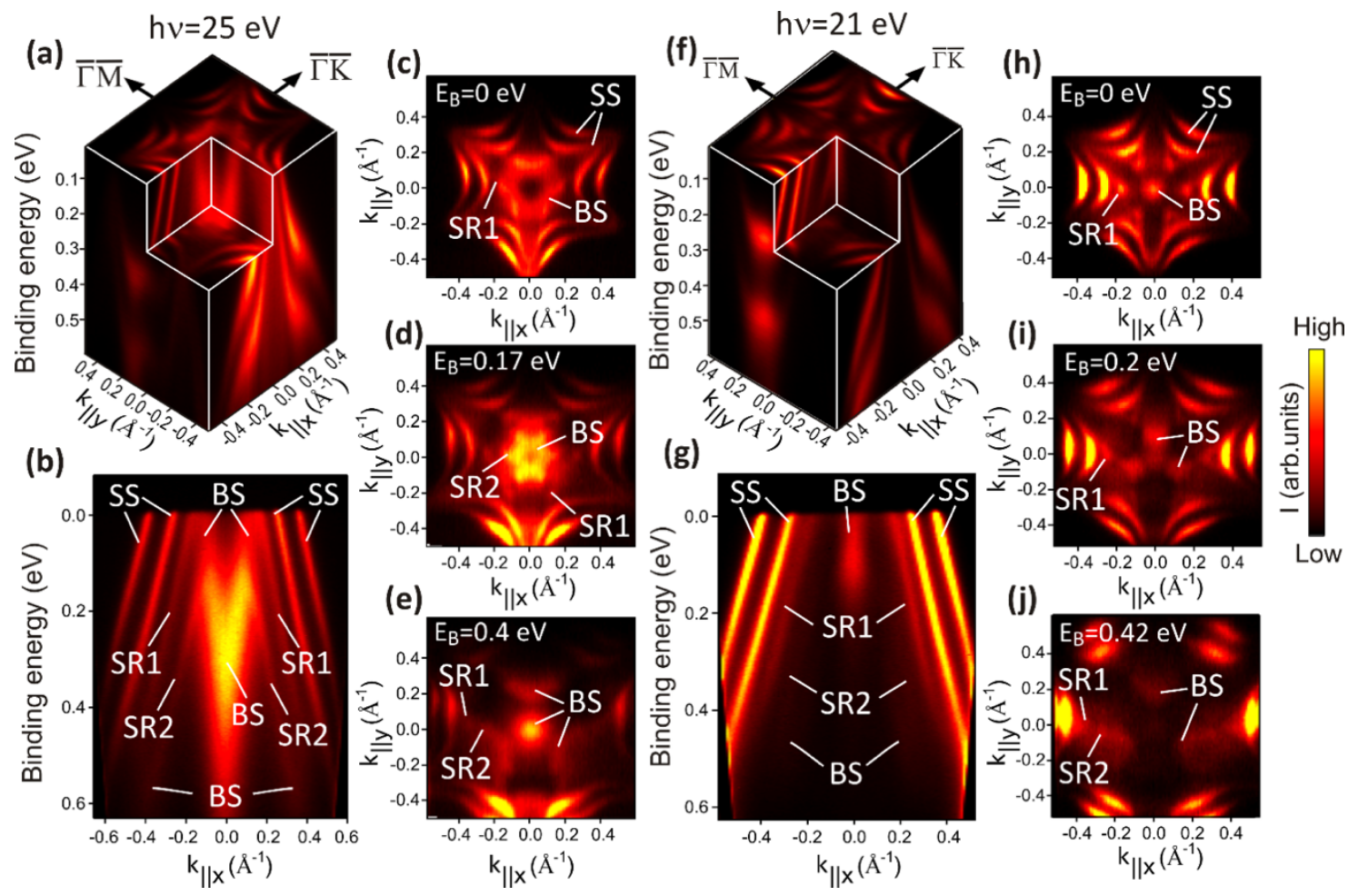

Figure 7. High-resolution ARPES dispersions of GeTe (111) bulk single crystal sampled with photon energies of (a)-(e) $25 \mathrm{eV}$ and (f)-(j) $21 \mathrm{eV}$ along different directions of the surface Brillouin zone. (a), (f) Full photoemission mapping around the $\bar{\Gamma}$ point. The $\bar{\Gamma}-\overline{\mathrm{K}}$ and $\bar{\Gamma}-\overline{\mathrm{M}}$ directions are oriented parallel to the in-plane electron wave vectors $\mathrm{k}_{\| \mathrm{x}}$ and $\mathrm{k}_{\| \mathrm{y}}$ respectively. (b), (g) Energymomentum dispersions along the $\bar{\Gamma}-\overline{\mathrm{K}}$ direction. Surface states, surface resonances and bulk states are denoted as SS, SR1 (SR2) and BS, respectively. (c)-(e), (h)-(j) Corresponding constant-energy surfaces extracted at different binding energies from the full photoemission mapping in (a), (f). 
The Rashba split surface state (denoted as SS in figures) can be seen crossing the Fermi level in the full photoemission mapping shown in Figs. $7 \mathrm{a}$ and $7 \mathrm{f}$, as well as in the corresponding energy-momentum dispersions taken along the $\bar{\Gamma}-\overline{\mathrm{K}}$ direction of the surface Brillouin zone (Figs. 7b,g).

The lack of photon energy dependence or $\mathrm{k}_{\mathrm{z}}$ dispersion of the surface state is also evident from the results shown in Fig. 8 (here, the $Z$ point in the fifth bulk Brillouin zone is observed at $h v=22 \mathrm{eV}$, which corresponds to $\mathrm{k}_{\mathrm{z}}=2.61 \AA^{-1}$ and an inner potential $\mathrm{U}_{0}=8.5 \mathrm{eV}$, as derived by taking into account the lattice constant $\mathrm{c}=5.98 \AA^{-1}$ along the $\mathrm{z}$ direction). In agreement with previous works, ${ }^{11-15,18,19}$ the surface state exhibits strongly warped snowflake contours as it can be seen in the constant-energy surfaces of Figs. 7ce and $7 \mathrm{~h}-\mathrm{j}$ for different binding energies. In particular, we derive a giant momentum splitting of $\Delta \mathrm{k}=0.12 \AA^{-1}$ as expected for the surface state associated to Te termination with an outwards polarization $P_{\text {out. }}{ }^{12}$
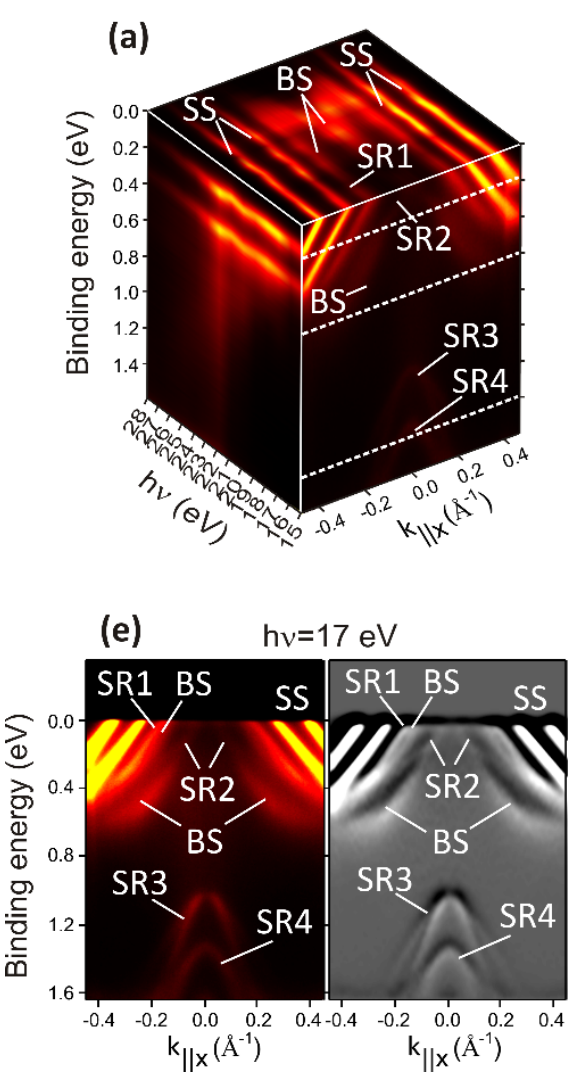
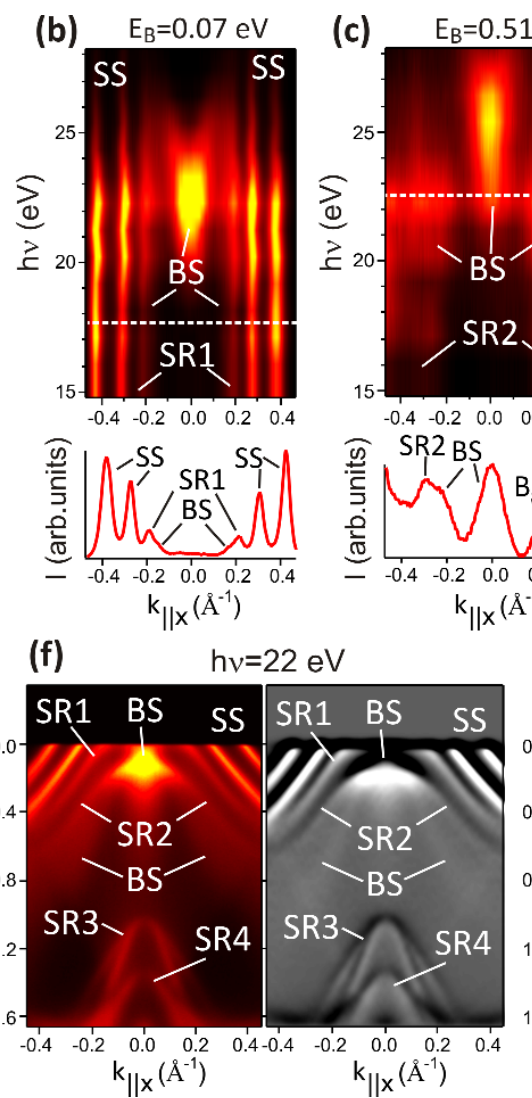
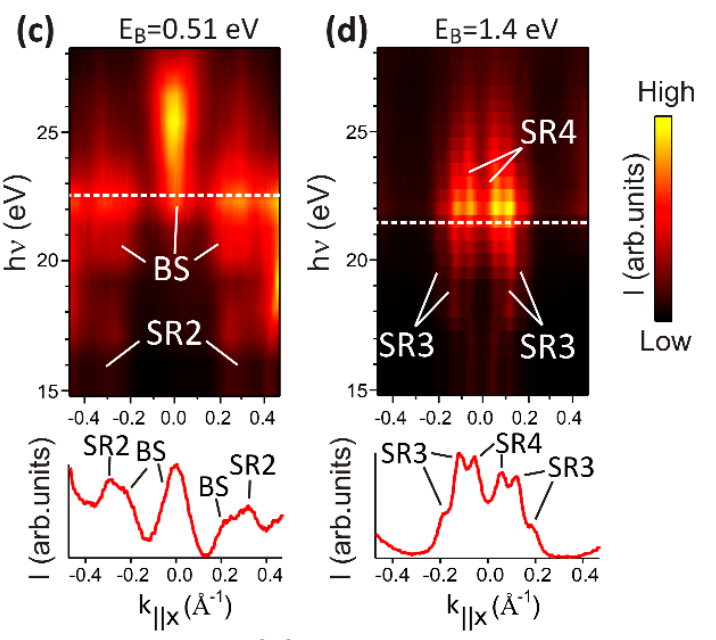

(g)

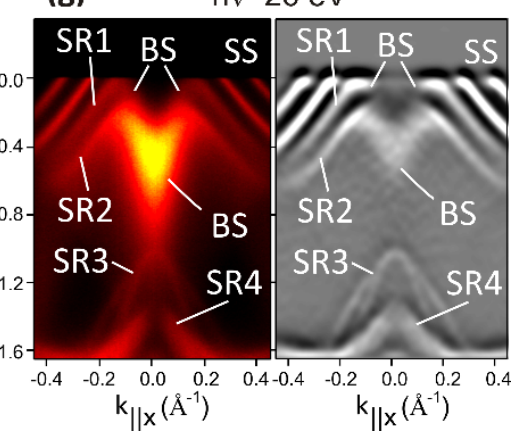

Figure 8. (a) Energy-momentum dispersions of surface states (SS), surface resonances (SR1-SR4) and bulk states (BS) as a function of photon energy. The $Z$ point of the bulk Brilluoin zone is located at $22 \mathrm{eV}$ photon energy. (b)-(d) Constant-energy surfaces extracted from (a) at binding energies of (b) $\mathrm{E}_{\mathrm{B}}=0.07 \mathrm{eV}$, (c) $0.51 \mathrm{eV}$ and (d) $1.4 \mathrm{eV}$. The bottom panels selectively show momentum-distribution curves (red solid lines) extracted at the energy positions corresponding to the horizontal white dashed lines displayed on the constant-energy surfaces. (e)-(g) Energymomentum dispersions extracted from (a) at photon energies of (e) $17 \mathrm{eV}$, (f) $22 \mathrm{eV}$, and (g) 26 $\mathrm{eV}$. In each panel, the second derivative of the photoemission intensity $\mathrm{d}^{2} \mathrm{I} / \mathrm{dE}^{2}$ is shown on the right.

At binding energies smaller than $0.6 \mathrm{eV}$, we also identify bulk-derived surface resonance states SR1 and SR2 which appear as weakly dispersing features with varying 
photon energy (Figs. 8a-c). Their intensity can also be resolved in the constant-energy surfaces of Figs. $7 \mathrm{c}-\mathrm{e}$ and $7 \mathrm{~h}-\mathrm{j}$ where they display six-fold symmetry and a strong overlap with the energy-momentum dispersion of pure bulk states (denoted as BS in figures).

While near the Fermi level and away from the $\bar{\Gamma}$ point we observe SR1 and SR2 states to split-off from pure bulk states, their separation in energy-momentum space with respect to dispersion of the bulk bands appears less pronounced than previously observed on single-terminated Te surfaces of MBE films. ${ }^{18}$ Moreover, due photoemission matrix-element effects, ${ }^{47,48}$ we observe a strong modulation in the relative intensity from surface resonances and bulk states as a function of photon energy (see e.g., Figs. 8b and 8c). In fact, because of the dispersing nature of the bulk bands with $\mathrm{k}_{\mathrm{Z}}$ and the influence of matrix elements, we are only able to resolve the separation between bulk bands and SR1 or SR2 states at off-normal wave vectors in some specific regions of the bulk Brillouin zone. These difficulties could be one of the reasons why SR1 and SR2 states were interpreted as pure bulk states in Refs. ${ }^{12,13}$. In our present data, the separation between SR1 and bulk states in the immediate vicinity of the Fermi level is well resolved at photon energies around $17 \mathrm{eV}$, while the same is true at photon energies around $22 \mathrm{eV}$ or near the $Z$ point for the separation between SR2 and bulk states at binding energies of $\sim 0.5 \mathrm{eV}$. For instance, both separations can be seen in the momentum-distribution curves shown in the bottom panels of Fig. $8 \mathrm{~b}$ and $8 \mathrm{c}$, where the intensity from bulk states forms shoulders on the sides corresponding to smaller $\mathrm{k}_{\| \mathrm{x}}$ wave vectors around the SR1 and SR2 peaks. Similarly, at $17 \mathrm{eV}$ photon energy, well-separated features corresponding to SR1, SR2 and bulk states are resolved near the Fermi level in the second derivative $\mathrm{d}^{2} \mathrm{I} / \mathrm{dE}^{2}$ of the photoemission intensity near $\mathrm{k}_{\| \mathrm{x}} \sim \pm 0.2 \AA^{-1}$ wave vectors (right panel in Fig. 8e). On the other hand, for $\left|\mathrm{k}_{|| \mathrm{x}}\right|<0.2 \AA^{-1}$ and at photon energies above $26 \mathrm{eV}$ (Fig. 8g), the intensity near the Fermi level is dominated by the contribution from bulk states which appear as intense and strongly dispersing features (compare e.g., Figs. 7b,g and 8e-g).

Overall, we find good agreement concerning the energy position and the dispersion of the bands with the DFT calculations of Ref. ${ }^{15}$, where SR1 and SR2 states are well reproduced in the calculated band dispersion of the Te-terminated surface. ${ }^{15}$ This is also the case at binding energies above $0.8 \mathrm{eV}$ in Fig. 8, where we find previously unmeasured features (labeled SR3 and SR4) without $\mathrm{k}_{\mathrm{Z}}$ dispersion (see e.g., Fig. 8d). These states appear as very narrow features with strong surface character exhibiting a Rashba splitting which can be resolved directly in Figs. 8d-g. For SR3 states, the momentum splitting is more pronounced and reaches values as large as $\Delta \mathrm{k}=0.07 \AA^{-1}$ in agreement with the theoretical prediction. ${ }^{15}$ It should be emphasized that these states are different than the pure bulk states appearing at binding energies above $1.5 \mathrm{eV}$ observed previously. ${ }^{14}$

Let us now come back to the dispersion of the bulk bands which is expected to be identical for both Te and Ge terminations except for the opposite spin polarization of the bands. ${ }^{15}$ Most recently, it was shown that the bulk bands of GeTe MBE films possessing a single Te termination are almost fully spin polarized, ${ }^{18}$ and that the magnitude of the spin polarization is similar for both bulk and surface bands. ${ }^{18}$ It was also established that the magnitude of the total spin polarization $\mathrm{P}_{\text {Tot }}=\left(\mathrm{P}_{\mathrm{x}}^{2}+\mathrm{P}_{\mathrm{y}}^{2}+\mathrm{P}_{\mathrm{z}}^{2}\right)^{1 / 2}$ is rather independent 
of the spin-orbit coupling strength, ${ }^{27}$ revealing the importance of the ferroelectric distortion, and thus the measured values of $\mathrm{P}_{\mathrm{Tot}}$ in GeTe directly represent the properties of the ground state. ${ }^{18}$ Keeping this in mind, we explored the magnitude and direction of the three-dimensional spin polarization vector for both surface and bulk states as shown in Fig. 9.

To this end, the spin polarization of the Rashba split surface state was acquired at $19.5 \mathrm{eV}$ photon energy and at off-normal wave vectors of $\mathrm{k}_{\| \mathrm{x}}=0.42 \AA^{-1}$ along the $\bar{\Gamma}-\overline{\mathrm{K}}$ direction (Fig. 9a) to reduce the influence of hybridization effects with SR1 states in the measured spin polarization. Under these conditions, we also observe the pure bulk states exhibiting a hole-like dispersion at binding energies above $1.5 \mathrm{eV}$ in Fig. 9a. ${ }^{14}$ We further checked the spin polarization at $28 \mathrm{eV}$ photon energy and at electron wave vectors $\mathrm{k}_{\| \mathrm{x}}=0.18 \AA^{-1}$ where the photoelectron intensity is dominated by the contribution of pure bulk states (Fig. 9b). The spin-resolved energy-distribution curves corresponding to the momentum cuts indicated by white dashed lines in Figs. 9a and 9b are displayed in Figs. $9 \mathrm{c}-\mathrm{e}$ and $9 \mathrm{f}-\mathrm{h}$, respectively. Here, the $\mathrm{S}_{\mathrm{y}}\left(\mathrm{S}_{\mathrm{x}}\right)$ and $\mathrm{P}_{\mathrm{y}}\left(\mathrm{P}_{\mathrm{x}}\right)$ components are perpendicular (parallel) to the electron momentum $\mathrm{k}_{\| \mathrm{x}}$ and lie on the surface plane, while the out-ofplane $\mathrm{S}_{\mathrm{z}}$ and $\mathrm{P}_{\mathrm{z}}$ components are perpendicular to the surface plane.
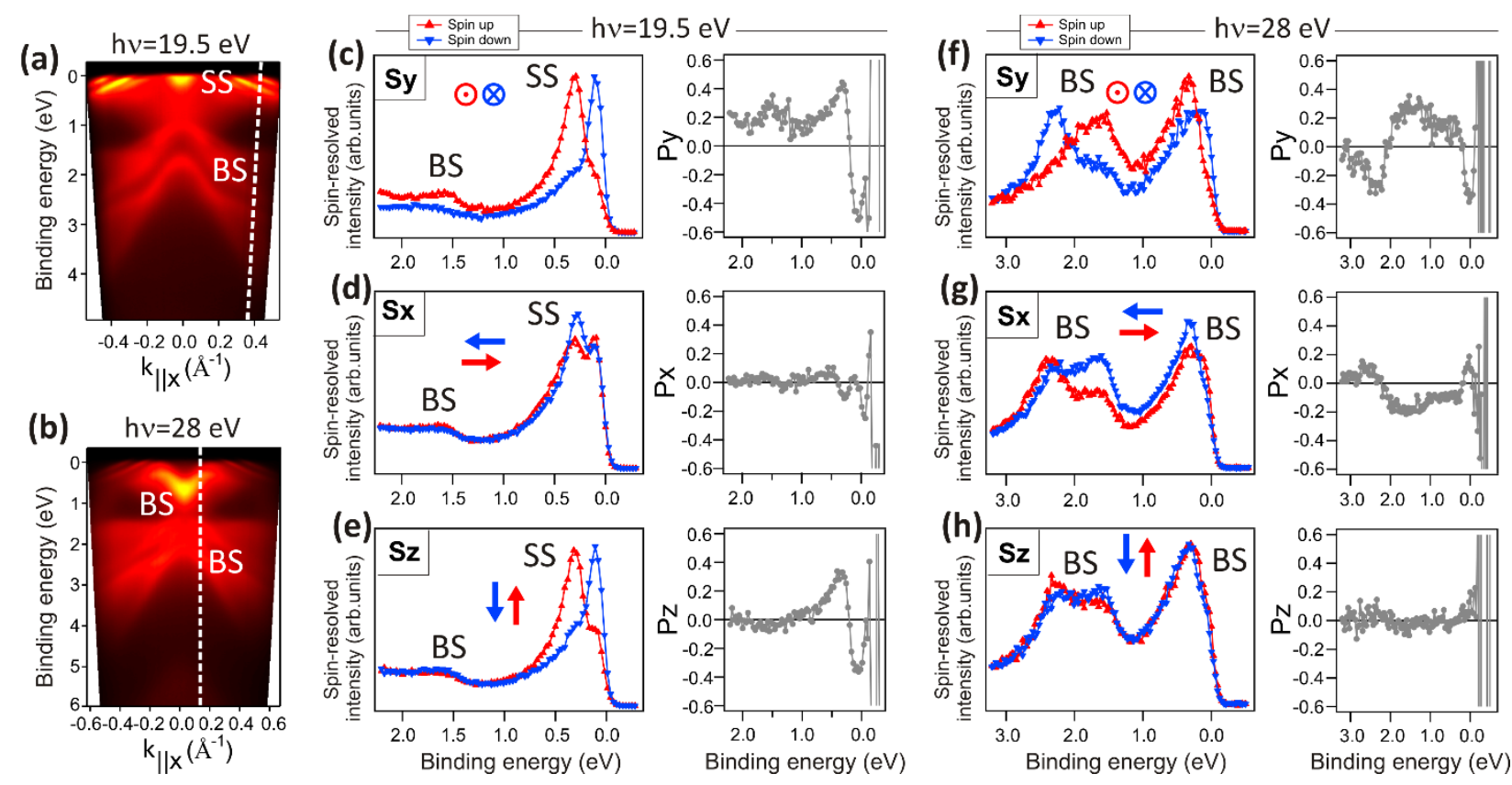

Figure 9. (a), (b) Energy-momentum dispersions of surface states (SS) and bulk states (BS) acquired in a wide binding energy range below the Fermi level and at photon energies of (a) 19.5 $\mathrm{eV}$ and (b) $28 \mathrm{eV}$. The different white dashed lines in (a) and (b) indicate the momentum cuts at which spin-resolved spectra in (c)-(e) and (f)-(h) have been taken, respectively. (c)-(h) Spin up (upwards red triangles) and spin down (downwards blue triangles) energy-distribution curves (left panels) and net spin polarizations (right panels) acquired along the three momentum axes at (c)(e) $19.5 \mathrm{eV}$ and (f)-(h) $28 \mathrm{eV}$ photon energy. The spin projections $\mathrm{S}_{\mathrm{y}}\left(\mathrm{S}_{\mathrm{x}}\right)$ and spin polarization $\mathrm{P}_{\mathrm{y}}$ $\left(\mathrm{P}_{\mathrm{x}}\right)$ are oriented perpendicular (parallel) to the electron momentum $\mathrm{k}_{\| \mathrm{x}}$, while the $\mathrm{S}_{z}$ and $\mathrm{P}_{z}$ components are perpendicular to the crystal surface. Spin down and negative spin polarization in (c) correspond to a clockwise rotation of electron spins as seen for the outer surface band, while in (d) to an additional out-of-plane canting of the spins in the direction towards the sample surface. 
Near the Fermi level, from Figs. 8 and 9b, we derive a momentum splitting of bulk states of about $\Delta \mathrm{k}=0.08 \AA^{-1}$. The energy splitting $\Delta \mathrm{E}$ of surface and bulk states in the spin-resolved data of Figs. $9 \mathrm{c}-\mathrm{e}$ and $9 \mathrm{f}-\mathrm{h}$ is about $210 \mathrm{meV}$ at $\mathrm{k}_{\mid \mathrm{x}}=0.42 \AA^{-1}$ and $90 \mathrm{meV}$ at $\mathrm{k}_{|| \mathrm{x}}=0.18 \AA^{-1}$, respectively. Taking into account these values, which are consistent with a linear scaling of the Rashba splitting, we derive a global Rashba parameter $\alpha_{R}=$ $2 \mathrm{E}_{0} / \mathrm{k}_{0}=2.8 \pm 0.8 \mathrm{eV} \times \AA^{-1}$, where $2 \mathrm{E}_{0} \sim \Delta \mathrm{E}$ and $\mathrm{k}_{0} \sim \Delta \mathrm{k} / 2$. This value of $\alpha_{\mathrm{R}}$ is close to the one predicted in recent bulk structure calculations. ${ }^{49}$

In Figs. 9c-e, it is seen that the spin vector component of the surface state along the $\mathrm{x}$ direction is negligible, while along the $\mathrm{y}$ direction it is oriented counterclockwise (clockwise) for the inner (outer) surface band, in agreement with the Rashba-type spin texture. ${ }^{12}$ Along the $z$ direction, we also observe non-zero spin polarization values which are consistent with the strong warping of the surface-state constant-energy contours (Figs. $7 \mathrm{c}-\mathrm{e}$ and $7 \mathrm{~h}-\mathrm{j}$ ), however, the values of $\mathrm{P}_{\mathrm{z}}$ are about two times larger than previously reported. ${ }^{14}$ In particular, we derive a maximum absolute spin polarization for the surface state bands of $\left|\mathrm{P}_{\mathrm{Tot}}^{\text {SS }}\right|=0.7 \pm 0.1$. Differently from this, for the bulk states at 19.5 $\mathrm{eV}$ in Figs. 9c-e the absolute spin polarization is substantially reduced and amounts to $\left|\mathrm{P}_{\mathrm{Tot}}^{\mathrm{BS}}\right|=0.3 \pm 0.1$. These lower values of the spin polarization of bulk states are quantitatively consistent with the results at $28 \mathrm{eV}$ in Figs. 9f-h from which we derive $\left|\mathrm{P}_{\mathrm{Tot}}^{\mathrm{BS}}\right|=0.34 \pm 0.1$. We do note that the reversed spin splitting of bulk states at binding energies above $1.5 \mathrm{eV}$ is consistent with their hole-like dispersion as established in previous DFT calculations. ${ }^{15}$ Connected to this, the appearance of a $\mathrm{S}_{\mathrm{x}}$ component in Fig. $9 \mathrm{~g}$ is likely related to the momentum and energy dependence in the relative contribution from $S$ and $p_{3 / 2}$ orbitals, however, this has a negligible influence on the magnitude of $\left|\mathrm{P}_{\text {Tot }}^{\text {BS }}\right| \cdot{ }^{18}$

The lower values of $\left|\mathrm{P}_{\mathrm{Tot}}^{\mathrm{BS}}\right|$ we observe are in contrast to the high degree of spin polarization of bulk states revealed for single Te-terminated film surfaces, ${ }^{18}$ pinpointing the crucial role of the multidomain structure revealed in the present work in reducing the values of $\left|\mathrm{P}_{\mathrm{Tot}}^{\mathrm{BS}}\right|$. Assuming that $\left|\mathrm{P}_{\mathrm{Tot}}^{\mathrm{SS}}\right| \approx\left|\mathrm{P}_{\mathrm{Tot}}^{\mathrm{BS}}\right|$ as shown in Ref. ${ }^{18}$, for a single Teterminated crystal surface, we would have expected values of $\left|\mathrm{P}_{\mathrm{Tot}}^{\mathrm{BS}}\right|$ of about 0.7 . If we now take into account our quantitative estimation of a 68/32 ratio in the relative contribution from $\mathrm{Te}$ and Ge terminations, respectively, we derive an expectation value of $\left|\mathrm{P}_{\mathrm{Tot}}^{\mathrm{BS}}\right| \approx 0.7 \cdot(0.68-0.32) \sim 0.26$ which is in quantitative agreement with our mean experimental value of $0.32 \pm 0.1$. We thus conclude that there is an intrinsic relationship between the opposite configurations of the ferroelectric polarization within the multidomain structure and the measured values of the bulk spin polarization. This unique correspondence is important especially considering that the large spin splitting of bulk states at the Fermi level and the electrical switchability of the spin polarization at the nanoscale could be exploited in novel functionalities of GeTe in non-volatile spintronic devices.

\section{Conclusions}

To summarize, using a complementary set of state-of-the-art experimental tools, we have investigated the atomic and electronic structure of GeTe (111) bulk single 
crystals grown by a direct solidification method. We have found a bulk domain structure consisting of inversion domains possessing two distinct types of boundaries i.e., nonplanar boundaries with a complex structure and flat Te-Te boundaries of the vdW type, both with dimensions at the atomic scale. The configuration of the inversion domains enables a cleavage plane along the [111] direction which proceeds through domains of different polarity, resulting in surface domains of both Te and Ge terminations. The lateral dimensions of the surface domains are in the range of tens to hundreds of nanometers, and both terminations reveal no reconstruction. Our results show good quantitative agreement between the calculated and experimental Ge $3 d$ and $\mathrm{Te} 4 d$ photoelectron diffraction patterns and corresponding core levels. The Te-terminated surface shows a relaxation of $\sim 3 \%$ and a surface core-level shift in the Te $4 d$ spectra associated to the potential due to the internal polarization of the top domains. The quantitative analysis reveals a 68/32 ratio in the relative contribution from surface domains of Te and Ge termination, respectively.

This result is consistent with our identification of spin-split surface states and surface resonances up to high binding energies in the band structure associated to the Te-termination. Finally, we have established an intrinsic quantitative relationship between the magnitude of the spin polarization of pure bulk states and the relative contribution of different terminations to the spin polarization. This correspondence is consistent with a reversal of the spin texture of the bulk Rashba bands for opposite configurations of the ferroelectric polarization within individual nanodomains.

The electrical reconfigurability of the spin texture at the nanoscale could be exploited in spintronic applications without the requirement of applied magnetic fields. There are different device proposals exploiting this possibility 2,5,50-52. For instance, storage of information could take place via the non-volatile ferroelectric material, i.e. by the reversibility of the ferroelectric polarization, and spin information transport could be exploited for computing capabilities via the lateral modulation of the spin polarization. In a broader context, our findings altogether are also important for other ferroelectric materials such as ferroelectric chalcogenides ${ }^{53}$, or even topological materials which are very close to a structural instability ${ }^{54,55}$ so that efficient tuning of hybridization and ionicity might render them ferroelectric.

\section{Methods}

GeTe single crystals ( $15 \mathrm{~g}$ ) were grown from the melt by direct solidification using a Te-enriched source containing 51 at. \% Te. For this $6 \mathrm{~N}$ pure elemental Ge and Te were waited in the corresponding ratio, melted in evacuated quartz cylindrical ampoules $(20 \mathrm{~mm} \times 40 \mathrm{~mm}$ ) and then air- quenched. Further prolonged annealing was performed isothermally at a temperature of $500^{\circ} \mathrm{C}$ for a period of more than 2 months. For LEED, STM, XPD, XPS, ARPES and spin-resolved ARPES measurements the GeTe crystal was cleaved in situ along the (111) plane under ultrahigh vacuum (UHV) conditions

and the base pressure of the experimental setups was better than $1 \times 10^{-10}$ mbar. The surfaces obtained after cleavage always exhibited sharp LEED patterns 
For all TEM measurements, the samples were prepared by focused ion beam (FIB) milling on $\mathrm{Cu}$ grids and the top surface layer of the FIB lamellas was protected by a carbon layer, followed by a platinum layer deposited with the electron beam and the ion beam, starting with the least invasive technique. The sample was thinned in five different steps with decreasing voltage (from 30 to $3 \mathrm{kV}$ ) and current (from 0.79 to $0.023 \mathrm{nA}$ ). The measurements were performed using FEI Titan aberration-corrected transmission electron microscopes operating at $300 \mathrm{kV}$. A double-tilt holder with $\mathrm{Cu}$ rings and tripod were used.

High-resolution STM studies were conducted using a commercial lowtemperature slider-type Createc microscope. All STM images were recorded at $77 \mathrm{~K}$ in constant-current mode using (001)-oriented single-crystalline tungsten tips, which were electrochemically etched in $2 \mathrm{M} \mathrm{NaOH}$ and sharpened in UHV using co-axial argon ion sputtering. ${ }^{56}$ In the STM experiments the bias voltage was applied to the sample with respect to the electrically grounded tip.

The XPD data were acquired at room temperature at the PEARL (X03DA) beamline $^{45}$ of the Swiss Light Source using linearly-polarized light. The direction perpendicular to the sample surface, the light polarization and the axis of the analyzer lens were oriented in the horizontal plane of the laboratory reference frame, while the entrance slit of the Scienta EW4000 electron analyzer was oriented vertically. PED maps were acquired by rotating the sample with polar angle steps of $1^{\circ}$. The acceptance azimuthal angle of the analyzer was restricted to $\pm 18^{\circ}$. The $2 \mathrm{D}$ electron detector allowed mapping of the angular distribution with azimuthal angle steps of less than $0.5^{\circ}$. The polar angle range was $80^{\circ}$. The calculation of XPD patterns was done with the EDAC code. ${ }^{57}$ $\mathrm{R}$-factor calculation, its minimization procedure and error estimation are described in Ref. ${ }^{45}$ We calculated the influence of kinetic energy variation on the fraction of $\mathrm{Ge}^{-}$ termination using EDAC code. In detail, we simulated patterns $555.0 \mathrm{eV}, 555.1 \mathrm{eV}$ and $556 \mathrm{eV}$. We found that the kinetic energy variation by $1 \mathrm{eV}$ results in Ge-termination fraction by $0.4 \% .1 \mathrm{eV}$ is less than photon energy error provided by Pearl beamline (see Ref. $\left.^{58}\right)$.

XPS measurements were performed at the Russian-German dipole beamline (RGBL) of the synchrotron light source BESSY-II at Helmholtz-Zentrum Berlin. The Ge $3 d$ and Te $4 d$ photoemission spectra were acquired with a hemispherical SPECS Phoibos 150 electron energy analyzer at normal emission geometry. The electron analyzer was calibrated using a clean Au reference sample, while the precise photon energy was determined using 2nd order reflections from the monochromator. XPS spectra for all elements were collected at similar kinetic energies (50-400 eV) to ensure the same surface sensitivity for both core-levels. The corresponding SCLS are calculated from the bulk state, which is monitored under the variation of surface sensitivity (i.e., by changing the photon energy), see Supporting information Fig. S7. All core-level spectra were fitted by Gaussian/Lorentzian convolution functions with simultaneous optimization of the background parameters using the UNIFIT 2014 software. ${ }^{59}$ The background was modeled using a combination of Shirley and Tougaard backgrounds.

XPS spectromicroscopy maps were obtained under ultrahigh vacuum conditions at a base pressure of $3 \times 10^{-10}$ mbar at the ESCA microscopy beamline of Elettra 
synchrotron source. Ge 3d and Te 4d maps were recorded at a photon energy of $652 \mathrm{eV}$ with a hemispherical electron analyzer equipped with a 48-channel photoelectron detector. By subtracting topography signal, we checked that photoemission intensity maps represent chemical contrast. The composition mapping was provided by focusing the X-ray beam to a $120 \mathrm{~nm}$ spot using a Fresnel zone plate. The spectra were acquired without a zone plate with a $70 \mu \mathrm{m}$ diameter spot size and the energy resolution of $250 \mathrm{meV}$. The cleanliness of the sample was carefully checked, and no contaminants were detected in the XPS spectra.

Angle-resolved photoemission (ARPES) and spin-resolved ARPES measurements were performed with linearly-polarized light at the U125-2-PGM RGBL Undulator and UE112-PGM2a beamlines of BESSY-II using the RGBL-2 and ARPES $1^{2}$ endstations, respectively. The end stations are equipped with hemispherical Scienta R4000 and R8000 analyzers, respectively, allowing parallel detection of multiple emission angles of the photoelectrons up to acceptance angles of $30^{\circ}$. The resolutions of ARPES measurements were set to $10 \mathrm{meV}$ (energy) and $0.3^{\circ}$ (angular). The RGBL-2 endstation is equipped with a combined detector which comprises a 2D channelplate for ARPES and a Mott-type spindetector, ${ }^{60}$ which was operated at $25 \mathrm{kV}$. The energy and angular resolutions of spinresolved ARPES measurements were $45 \mathrm{meV}$ and $0.75^{\circ}$, respectively. For positioning and angular orientation of samples in the experiments, a LHe-cooled 6 -axis cryomanipulator was operated either at $\mathrm{T}=40 \mathrm{~K}$ or at $25 \mathrm{~K}$.

Theoretical modeling of the surface performed within the DFT approach using the PW-GGA method. ${ }^{61}$ Plane-wave basis sets with a cutoff energy of $\mathrm{E}_{\text {cut }}=400 \mathrm{eV}$ and the projector augmented-wave method ${ }^{62}$ were employed as implemented in the Vienna $a b$ initio Simulation Package (VASP). ${ }^{63-65}$ Surface slabs were constructed as hexagonal (1 x1 $\mathrm{x} 4$ ) supercells. Geometry was fully optimized. Structural relaxations were performed until residual forces fell below $10^{-3} \mathrm{eV} \cdot \AA^{-1}$. Stopping criterion for electronic SCF optimization was a difference in the total energy of less than $10^{-4} \mathrm{eV}$ between two consecutive iterations. Calculations were performed using $\Gamma$-centered Monkhorst-Pack grid of $2 \times 2 \times 1$ points. Ge $3 \mathrm{~d}^{10} 4 \mathrm{~s}^{2} 4 \mathrm{p}^{2}$, Te $5 \mathrm{~s}^{2} 5 \mathrm{p}^{4}$ orbitals were included in the valence electron shell. Core-level shifts were calculated in the initial-state approximation as variation of electrostatic potentials at the atomic centers. ${ }^{66}$ The difference between the potentials for the atoms of central layer of the slab ("bulk") and for each layer of Te-and Ge-terminated surface were calculated. Theoretical Ge $3 d$ and Te $4 d$ spectra are composed by several peaks corresponding to each atomic layer of the slab for $\mathrm{Ge}^{-}$or $\mathrm{Te}^{-}$terminations in a proportion defined by the electron kinetic energy (surface sensitivity) and by including experimental broadening. The spectra obtained for different terminations were added and their ratio was optimized to quantitatively describe the experimental XPS spectra.

\section{Acknowledgements}

Financial support from the Russian Science Foundation (RSF) under Grant No. 1942-06303 and from the Impuls-und Vernetzungsfonds der Helmholtz Gemeinschaft under Grant No. HRSF-0067 (Helmholtz-Russia Joint Research Group) is gratefully acknowledged. J.H. and C.C. acknowledge support through the BOF Grant No. 31445. A.C., 
B.W. and K.Z. wish to acknowledge an Erasmus+ mobility grant (2017-1-IE02-KA107000538 \& 2017-1-IE02-KA107-000538). A.S.F. acknowledges the travel support of the German-Russian Interdisciplinary Science Center (G-RISC) funded by the German Federal Foreign Office via the German Academic Exchange Service (DAAD). We thank Helmholtz-Zentrum Berlin for granting access to the beamlines and for provision of beamtime at the synchrotron radiation source BESSY-II in Berlin. We acknowledge the Paul Scherrer Institut for provision of synchrotron radiation beamtime at the PEARL beamline of the Swiss Light Source. We gratefully acknowledge Moscow State University for providing access to the Lomonosov supercomputer.

\section{Supporting information contents}

More examples of HAADF-STEM and STM images, photon energy dependence of Ge $3 d$ and Te $4 d$ spectra, as well as details of XPD and SPEM measurements, can be found in Supplementary Information section.

\section{References}

(1) Datta, S.; Das, B. Electronic Analog of the Electro-optic Modulator. Appl Phys Lett 1990, 56 (7), 665-667.

(2) Žutić, I.; Fabian, J.; Sarma, S. D. Spintronics: Fundamentals and Applications. Rev Mod Phys 2004, $76(2), 323-410$.

(3) Awschalom, D.; Samarth, N. Spintronics without Magnetism. Phys 2009, 2, 50.

(4) Cahay, M. Closer to an All-Electric Device. Nat Nanotechnol 2015, 10 (1), 21-22.

(5) Picozzi, S. Ferroelectric Rashba Semiconductors as a Novel Class of Multifunctional Materials. Aip Conf Proc 2014, 2, 10.

(6) Pawley, G. S.; Cochran, W.; Cowley, R. A.; Dolling, G. Diatomic Ferroelectrics. Phys Rev Lett 1966, 17 (14), 753-755.

(7) Steigmeier, E. F.; Harbeke, G. Soft Phonon Mode and Ferroelectricity in GeTe. Solid State Commun 1970, 8 (16), 1275-1279.

(8) Li, J.; Zhang, X.; Chen, Z.; Lin, S.; Li, W.; Shen, J.; Witting, I. T.; Faghaninia, A.; Chen, Y.; Jain, A.; Chen, L.; Snyder, G. J.; Pei, Y. Low-Symmetry Rhombohedral GeTe Thermoelectrics. Joule 2018, 2 (5), 976-987.

(9) Bayikadi, K. S.; Sankar, R.; Wu, C. T.; Xia, C.; Chen, Y.; Chen, L.-C.; Chen, K.-H.; Chou, F.-C. Enhanced Thermoelectric Performance of GeTe through in Situ Microdomain and GeVacancy Control. J Mater Chem A 2019, 7 (25), 15181-15189.

(10) Yamada, N.; Ohno, E.; Nishiuchi, K.; Akahira, N.; Takao, M. Rapid-phase Transitions of GeTe-Sb 2 Te 3 Pseudobinary Amorphous Thin Films for an Optical Disk Memory. J Appl Phys 1991, 69 (5), 2849-2856. 
(11) Sante, D. D.; Barone, P.; Bertacco, R.; Picozzi, S. Electric Control of the Giant Rashba Effect in Bulk GeTe. Adv Mater 2012, 25 (4), 509-513.

(12) Liebmann, M.; Rinaldi, C.; Sante, D. D.; Kellner, J.; Pauly, C.; Wang, R. N.; Boschker, J. E.; Giussani, A.; Bertoli, S.; Cantoni, M.; Baldrati, L.; Asa, M.; Vobornik, I.; Panaccione, G.; Marchenko, D.; Sánchez-Barriga, J.; Rader, O.; Calarco, R.; Picozzi, S.; Bertacco, R.;

Morgenstern, M. Giant Rashba-Type Spin Splitting in Ferroelectric GeTe(111). Adv Mater 2016, $28(3), 560-565$.

(13) Elmers, H. J.; Wallauer, R.; Liebmann, M.; Kellner, J.; Morgenstern, M.; Wang, R. N.; Boschker, J. E.; Calarco, R.; Sánchez-Barriga, J.; Rader, O.; Kutnyakhov, D.; Chernov, S. V.; Medjanik, K.; Tusche, C.; Ellguth, M.; Volfova, H.; Borek, St.; Braun, J.; Minár, J.; Ebert, H.; Schönhense, G. Spin Mapping of Surface and Bulk Rashba States in Ferroelectric $\alpha-G e T e(111)$ Films. Phys Rev B 2016, 94 (20), 201403.

(14) Krempaský, J.; Volfová, H.; Muff, S.; Pilet, N.; Landolt, G.; Radović, M.; Shi, M.; Kriegner, D.; Holý, V.; Braun, J.; Ebert, H.; Bisti, F.; Rogalev, V.; Strocov, V.; Springholz, G.; Minár, J.; Dil, J. Disentangling Bulk and Surface Rashba Effects in Ferroelectric $\alpha$-GeTe. Phys Rev B 2016, 94 (20), 205111.

(15) Rinaldi, C.; Varotto, S.; Asa, M.; Sławińska, J.; Fujii, J.; Vinai, G.; Cecchi, S.; Sante, D. D.; Calarco, R.; Vobornik, I.; Panaccione, G.; Picozzi, S.; Bertacco, R. Ferroelectric Control of the Spin Texture in GeTe. Nano Lett 2018, 18 (5), 2751-2758.

(16) Lencer, D.; Salinga, M.; Grabowski, B.; Hickel, T.; Neugebauer, J.; Wuttig, M. A Map for Phase-Change Materials. Nat Mater 2008, 7 (12), 972-977.

(17) Lucovsky, G.; White, R. M. Effects of Resonance Bonding on the Properties of Crystalline and Amorphous Semiconductors. Phys Rev B 1973, 8 (2), 660-667.

(18) Krempaský, J.; Fanciulli, M.; Nicolaï, L.; Minár, J.; Volfová, H.; Caha, O.; Volobuev, V. V.; Sánchez-Barriga, J.; Gmitra, M.; Yaji, K.; Kuroda, K.; Shin, S.; Komori, F.; Springholz, G.; Dil, J. H. Fully Spin-Polarized Bulk States in Ferroelectric GeTe. Phys Rev Res 2020, 2 (1), 013107.

(19) Krempaský, J.; Muff, S.; Minár, J.; Pilet, N.; Fanciulli, M.; Weber, A. P.; Guedes, E. B.; Caputo, M.; Müller, E.; Volobuev, V. V.; Gmitra, M.; Vaz, C. A. F.; Scagnoli, V.; Springholz, G.; Dil, J. H. Operando Imaging of All-Electric Spin Texture Manipulation in Ferroelectric and Multiferroic Rashba Semiconductors. Phys Rev X 2018, 8 (2), 021067..

(20) Polking, M. J.; Han, M.-G.; Yourdkhani, A.; Petkov, V.; Kisielowski, C. F.; Volkov, V. V.; Zhu, Y.; Caruntu, G.; Alivisatos, A. P.; Ramesh, R. Ferroelectric Order in Individual NanometreScale Crystals. Nat Mater 2012, 11 (8), 700-709.

(21) Nelson, C. T.; Gao, P.; Jokisaari, J. R.; Heikes, C.; Adamo, C.; Melville, A.; Baek, S.-H.; Folkman, C. M.; Winchester, B.; Gu, Y.; Liu, Y.; Zhang, K.; Wang, E.; Li, J.; Chen, L.-Q.; Eom, C.-B.; Schlom, D. G.; Pan, X. Domain Dynamics During Ferroelectric Switching. Science 2011, 334 (6058), 968-971.

(22) Nukala, P.; Ren, M.; Agarwal, R.; Berger, J.; Liu, G.; Johnson, A. T. C.; Agarwal, R. Inverting Polar Domains via Electrical Pulsing in Metallic Germanium Telluride. Nat Commun 2017, 8 (1), 15033. 
(23) Shin, Y.-H.; Grinberg, I.; Chen, I.-W.; Rappe, A. M. Nucleation and Growth Mechanism of Ferroelectric Domain-Wall Motion. Nature 2007, 449 (7164), 881-884.

(24) Snykers, M.; Delavignette, P.; Amelinckx, S. The Domain Structure of GeTe as Observed by Electron Microscopy. Mater Res Bull 1972, 7 (8), 831-839.

(25) Lee, H. S.; Kim, B.-S.; Cho, C.-W.; Oh, M.-W.; Min, B.-K.; Park, S.-D.; Lee, H.-W.

Herringbone Structure in GeTe-Based Thermoelectric Materials. Acta Mater 2015, 91, 83-90.

(26) Lupascu, D. C. Fatigue in Ferroelectric Ceramics and Related Issues. 2004.

(27) Krempaský, J.; Springholz, G.; Minár, J.; Dil, J. H. $\alpha$-GeTe and (GeMn)Te Semiconductors: A New Paradigm for Spintronics. 2018, 020026.

(28) Schubert, K.; Fricke, H. Notizen: Kristallstruktur von GeTe. Zeitschrift Für Naturforschung 1951, 6 (12), 781-782.

(29) Backer, A. D.; Bos, K. H. W. van den; Broek, W. V. den; Sijbers, J.; Aert, S. V. StatSTEM: An Efficient Approach for Accurate and Precise Model-Based Quantification of Atomic Resolution Electron Microscopy Images. Ultramicroscopy 2016, 171, 104-116.

(30) Wojdyr, M. Fityk : A General-Purpose Peak Fitting Program. J Appl Crystallogr 2010, 43 (5), 1126-1128.

(31) Hahn, Th.; Klapper, H. International Tables for Crystallography. 2006, 393-448.

(32) Kalra, G.; Murugavel, S. The Role of Atomic Vacancies on Phonon Confinement in $\alpha$ GeTe. Aip Adv 2015, 5 (4), 047127.

(33) Feng, J.; Xu, M.; Wang, X.; Lin, Q.; Cheng, X.; Xu, M.; Tong, H.; Miao, X. Gold Fillings Unravel the Vacancy Role in the Phase Transition of GeTe. Appl Phys Lett 2018, 112 (7), 071902.

(34) Tong, F.; Miao, X. S.; Wu, Y.; Chen, Z. P.; Tong, H.; Cheng, X. M. Effective Method to Identify the Vacancies in Crystalline GeTe. Appl Phys Lett 2010, 97 (26), 261904.

(35) Sist, M.; Kasai, H.; Hedegaard, E. M. J.; Iversen, B. B. Role of Vacancies in the HighTemperature Pseudodisplacive Phase Transition in GeTe. Phys Rev B 2018, 97 (9), 094116.

(36) Yashina, L. V.; Sánchez-Barriga, J.; Scholz, M. R.; Volykhov, A. A.; Sirotina, A. P.; Neudachina, V., S.; Tamm, M. E.; Varykhalov, A.; Marchenko, D.; Springholz, G.; Bauer, G.; Knop-Gericke, A.; Rader, O. Negligible Surface Reactivity of Topological Insulators Bi 2 Se 3 and Bi 2 Te 3 towards Oxygen and Water. Acs Nano 2013, 7 (6), 5181-5191.

(37) Sirotina, A. P.; Callaert, C.; Volykhov, A. A.; Frolov, A. S.; Sánchez-Barriga, J.; KnopGericke, A.; Hadermann, J.; Yashina, L. V. Mechanistic Studies of Gas Reactions with Multicomponent Solids: What Can We Learn By Combining NAP XPS and Atomic Resolution STEM/EDX? J Phys Chem C 2019, 123 (43), 26201-26210.

(38) Anderson, J. S.; Browne, J. M.; Cheetam, A. K.; Dreele, R. V.; Hutchison, J. L.; Lincoln, F. J.; Bevan, D. J. M.; Straehle, J. Point Defects and Extended Defects in Niobium Oxides. Nature 1973, 243 (5402), 81-83. 
(39) Nonstoichiometric Oxides; Sørensen, O. T., Ed.; 1981.

(40) Chaika, A. N.; Nazin, S. S.; Semenov, V. N.; Bozhko, S. I.; Lübben, O.; Krasnikov, S. A.; Radican, K.; Shvets, I. V. Selecting the Tip Electron Orbital for Scanning Tunneling Microscopy Imaging with Sub-Ångström Lateral Resolution. Epl Europhys Lett 2010, 92 (4), 46003.

(41) Grushko, V.; Lübben, O.; Chaika, A. N.; Novikov, N.; Mitskevich, E.; Chepugov, A.; Lysenko, O.; Murphy, B. E.; Krasnikov, S. A.; Shvets, I. V. Atomically Resolved STM Imaging with a Diamond Tip: Simulation and Experiment. Nanotechnology 2014, 25 (2), 025706.

(42) Deringer, V. L.; Lumeij, M.; Dronskowski, R. Ab Initio Modeling of $\alpha-G e T e(111)$ Surfaces. J Phys Chem C 2012, 116 (29), 15801-15811.

(43) Fadley, C. S. Angle-Resolved x-Ray Photoelectron Spectroscopy. Prog Surf Sci 1984, 16 (3), 275-388.

(44) Kuznetsov, M. V.; Ogorodnikov, I. I.; Usachov, D. Yu.; Laubschat, C.; Vyalikh, D. V.; Matsui, F.; Yashina, L. V. Photoelectron Diffraction and Holography Studies of 2D Materials and Interfaces. J Phys Soc Jpn 2018, 87 (6), 061005.

(45) Usachov, D. Yu.; Tarasov, A. V.; Bokai, K. A.; Shevelev, V. O.; Vilkov, O. Yu.; Petukhov, A. E.; Rybkin, A. G.; Ogorodnikov, I. I.; Kuznetsov, M. V.; Muntwiler, M.; Matsui, F.; Yashina, L. V.; Laubschat, C.; Vyalikh, D. V. Site- and Spin-Dependent Coupling at the Highly Ordered h-BN/Co(0001) Interface. Phys Rev B 2018, 98 (19), 195438.

(46) Yashina, L. V.; Püttner, R.; Neudachina, V. S.; Zyubina, T. S.; Shtanov, V. I.; Poygin, M. V. X-Ray Photoelectron Studies of Clean and Oxidized $\alpha$-GeTe(111) Surfaces. J Appl Phys 2008, 103 (9), 094909.

(47) Angle-Resolved Photoemission, Theory, and Current Applications; Kevan, D., S., Eds.; Studies in Surface Science and Catalysis; Elsevier: Amsterdam, 1991; Vol. 72.

(48) Very High Resolution Photoelectron Spectroscopy; Hüfner, S., Ed.; Springer-Verlag: Berlin Heidelberg, 2007.

(49) Wang, H.; Gopal, P.; Picozzi, S.; Curtarolo, S.; Nardelli, M. B.; Sławińska, J. Spin Hall Effect in Prototype Rashba Ferroelectrics GeTe and SnTe. Npj Comput Mater 2020, 6 (1), 7.

(50) Rabe, K. M.; Ghosez, P. Physics of Ferroelectrics, A Modern Perspective. 2007, 117-174.

(51) Phase Change Materials. 2009.

(52) Manchon, A.; Koo, H. C.; Nitta, J.; Frolov, S. M.; Duine, R. A. New Perspectives for Rashba Spin-Orbit Coupling. Nat Mater 2015, 14 (9), 871-882.

(53) Littlewood, P. B. The Crystal Structure of IV-VI Compounds. I. Classification and Description. J Phys C Solid State Phys 2000, 13 (26), 4855.

(54) Kooi, B. J.; Noheda, B. Ferroelectric Chalcogenides-Materials at the Edge. Science 2016, 353 (6296), 221-222. 
(55) Mandal, P. S.; Springholz, G.; Volobuev, V. V.; Caha, O.; Varykhalov, A.; Golias, E.; Bauer, G.; Rader, O.; Sánchez-Barriga, J. Topological Quantum Phase Transition from Mirror to Time Reversal Symmetry Protected Topological Insulator. Nat Commun 2017, 8 (1), 968.

(56) Chaika, A. N.; Orlova, N. N.; Semenov, V. N.; Postnova, E. Yu.; Krasnikov, S. A.; Lazarev, M. G.; Chekmazov, S. V.; Aristov, V. Yu.; Glebovsky, V. G.; Bozhko, S. I.; Shvets, I. V. Fabrication of [001]-Oriented Tungsten Tips for High Resolution Scanning Tunneling Microscopy. Sci Rep-uk 2014, 4 (1), 3742.

(57) Abajo, F. J. G. de; Hove, M. A. V.; Fadley, C. S. Multiple Scattering of Electrons in Solids and Molecules: A Cluster-Model Approach. Phys Rev B 2001, 63 (7), 075404.

(58) Muntwiler, M.; Zhang, J.; Stania, R.; Matsui, F.; Oberta, P.; Flechsig, U.; Patthey, L.; Quitmann, C.; Glatzel, T.; Widmer, R.; Meyer, E.; Jung, T. A.; Aebi, P.; Fasel, R.; Greber, T. Surface Science at the PEARL Beamline of the Swiss Light Source. J Synchrotron Radiat 2017, 24 (1), 354-366.

(59) Unifit 2014. https://home.uni-leipzig.de/unifit/new.htm https://home.unileipzig.de/unifit/new.htm.

(60) Burnett, G. C.; Monroe, T. J.; Dunning, F. B. High-efficiency Retarding-potential Mott Polarization Analyzer. Rev Sci Instrum 1994, 65 (6), 1893-1896.

(61) Perdew, J. P.; Burke, K.; Ernzerhof, M. Generalized Gradient Approximation Made Simple. Phys Rev Lett 1996, 77 (18), 3865-3868.

(62) Blöchl, P. E. Projector Augmented-Wave Method. Phys Rev B 1994, 50 (24), 17953-17979.

(63) Kresse, G.; Hafner, J. Ab Initio Molecular Dynamics for Liquid Metals. Phys Rev B 1993, 47 (1), 558-561.

(64) Kresse, G.; Furthmüller, J. Efficiency of Ab-Initio Total Energy Calculations for Metals and Semiconductors Using a Plane-Wave Basis Set. Comp Mater Sci 1996, 6 (1), 15-50.

(65) Kresse, G.; Joubert, D. From Ultrasoft Pseudopotentials to the Projector Augmented-Wave Method. Phys Rev B 1998, 59 (3), 1758-1775.

(66) Yashina, L. V.; Zyubina, T. S.; Püttner, R.; Zyubin, A. S.; Shtanov, V. I.; Tikhonov, E. V. A Combined Photoelectron Spectroscopy and Ab Initio Study of the Adsorbate System O 2 /PbTe(001) and the Oxide Layer Growth Kinetics. J Phys Chem C 2008, 112 (50), 19995-20006. 